\title{
Nanogel Functionalization: A Versatile Approach To Meet the Challenges of Drug and Gene Delivery
}

\author{
Emanuele Mauri, ${ }^{\dagger} \neq$ Giuseppe Perale, ${ }^{\S}$ and Filippo Rossi* ${ }^{* \dagger}$ (i) \\ ${ }^{\dagger}$ Department of Chemistry, Materials and Chemical Engineering “Giulio Natta”, Politecnico di Milano, via Mancinelli 7, 20131 \\ Milan, Italy \\ ${ }^{\ddagger}$ Department of Engineering, Tissue Engineering and Chemistry for Engineering Unit, Università Campus Bio-Medico di Roma, via \\ Alvaro del Portillo 21, 00128 Rome, Italy \\ ${ }^{\S}$ Biomaterials Laboratory, Institute for Mechanical Engineering and Materials Technology, University of Applied Sciences and Arts \\ of Southern Switzerland, via Cantonale 2C, Galleria 2, 6928 Manno, Switzerland
}

\begin{abstract}
The design of nanostructures carrying therapeutic molecules represents the new frontier in the biological and medical fields. In particular, researchers have identified nanogels as promising tools in response to the critical issues of intracellular delivery: because of their peculiar properties, including swelling behavior, nanogels are able to cross the cellular membrane (clathrin-mediated endocytosis, caveolin-mediated endocytosis, phagocytosis, and macropinocytosis) and release their cargo in the cytosol, avoiding the activation of immune responses. However, only the combination of different starting polymers is not enough for the development of selective and targeted nanocarriers. The orthogonal functionalization of polymer chains with different chemical groups allows grafting of specific moieties and conjugating biomolecules, such as peptides, proteins, and growth factors, to give rise to nanogels that are not only more attractive but also more efficient in drug and gene delivery, with the ability

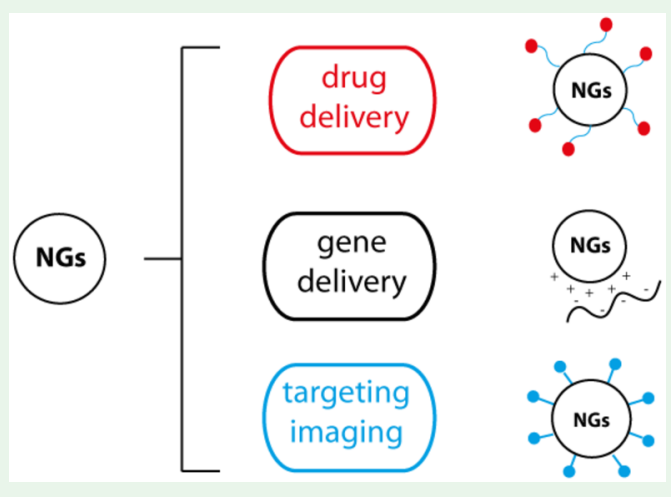
to reach specific sites and trigger selective therapies. Moreover, the functionalization techniques offer the opportunity to improve load protection until the desired area is reached and synthesize nanosystems that are sensitive to biological stimuli. This review discusses the key role of nanogel functionalization to design promising nanonetworks for drug and gene delivery, highlighting how modification of the polymer meets the challenges of biomolecule transport, and summarizes the most recent results recorded in these fields. A brief mention is also accorded to the functionalization approach to produce detectable nanogels in vitro and in vivo, ensuring their traceability over time.
\end{abstract}

KEYWORDS: colloids, drug delivery, gene delivery, nanogels, polymers

\section{INTRODUCTION}

Nowadays, nanotechnology advancements in the medical and biological fields have led to the development of innovative and alternative approaches for therapies and pharmacological and diagnostic investigations in the scientific, social, and ethical challenges. $^{1-3}$ The local manipulation of matter on the atomic and molecular scale concerns material structures and components that have to exhibit novel and significantly improved physical, chemical, and biological properties. ${ }^{4,5}$ In this framework, the needs for targeted drug delivery systems, cell-compatible scaffolds, bone replacements, tracking and imaging techniques, and medical tools for the clinical treatment of tumors, cancer, or other neurodegenerative diseases have moved researchers to the fabrication of many types of nanomaterials, including liposomes, micelles, polymeric nanoparticles, carbon nanotubes, metallic nanostructures, and quantum dots. Among them, nanogels have gained increasing interest because of their potential in medicine, bioengineering, and diagnostic applications. ${ }^{6,7}$ The unique properties of nanogels are closely correlated to the chance of fine-tuning their porosity, hydrophilic nature, stability, size, and charge by varying the chemical composition or grafting of additional functionalities. These nanosystems are also characterized by improved colloidal stability compared with surfactant micelles due to lower rates of dissociation/degradation, longer retention of loaded cargo (thanks to the large surface area to volume ratio), and their peculiar ability to retain a high water content that enhances dispersion stability. In particular, because of the swelling behavior and the ability to encapsulate and protect biomolecules, nanogels have grown more attractive for drug delivery and gene delivery because they can reach specific sites and trigger selective responses. ${ }^{8}$ In drug delivery, the pivotal aim is controlled drug release within the therapeutic concentration range through the use of a vehicle that is able to counteract the risk of under- or overdosing, which could trigger potential side effects and toxicity. Engineering of nanogels can achieve

Received: September 25, 2018

Accepted: November 14, 2018

Published: November 14, 2018 

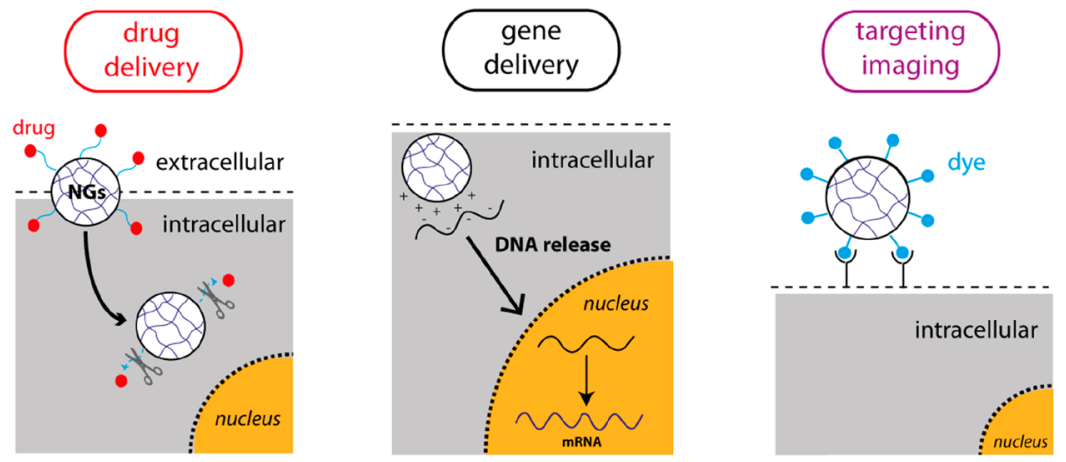

Figure 1. Schematic representation of nanogels in biological applications.

improved control over the release kinetics because of their small size and higher surface area, and they can be internalized by cells, surmounting the selective control of the cell membrane and releasing the drug in response to intracellular cues.

In gene delivery, the crucial point is related to the transport of genetic material such as DNA or RNA into host cells to regulate the proteins or genome expression in cancer therapies and in neurological disorders in order to mitigate the degeneration. Nanogels can be designed to load nucleic acids, circulate in the human body, and target diseases at the site of disorder. The smart combination of different polymers to improve the nanogel performance in the biological environment is generally the first step to assess drug carriers or selective material-cell interactions. However, the resulting biomaterials, despite the promising physicomechanical properties and the wide variety of optimized configurations, are composed of natural or synthetic polymers that, even if they are biocompatible, have some restrictions due to their proper chemical structures. Indeed, there is a high necessity to insert additional functionality that comes from the fact that not all desirable functional groups are available in polymer chains. Modification of the polymer backbone through different chemoselective and orthogonal strategies allows the grafting of chemical functional groups or compounds to design a more attractive and suitable nanosystem. ${ }^{9,10}$ Two main approaches have been developed to face the challenges in drug and gene delivery: functionalization of the polymers before nanogel synthesis (prefunctionalization) and introduction of bioresponsive moieties after nanogel synthesis (postfunctionalization). The review aims to present the recent examples of nanogel functionalization to link specific chemical groups, proteins, peptides, or target molecules that make them suitable for the drug and gene delivery domain. The discussion is focused on the synthesis of the cross-linking network using the modified materials and the subsequent effects in terms of stimuli response, bioconjugation, and encapsulation of bioactive compounds. Moreover, the traceability of the nanoparticles represents another significant aspect: their biodistribution within the cytosol or during circulation in the blood, their storage in tissues or in organs, and their degradation times can be monitored using imaging or tracking techniques. The principle of postpolymerization modification is applicable in this context to design traceable nanotools for disease detection, diagnosis, and treatment monitoring. The last part of this review is focused on the orthogonal polymer modification for imaging techniques. A schematic representation of the three considered topics is shown in Figure 1: nanogels for drug delivery, gene delivery, and targeting imaging.

\section{KEY PHYSICAL FEATURES OF NANOGELS}

Nanogels are composed of chemically or physically cross-linked polymers rearranged to form a three-dimensional spherical-like network. Recent advancements in experimental procedures also provide the opportunity of specific shape in the fabrication of nanosystems. ${ }^{11-13}$ The term "nanogel" has its source in the research of Vinogradov and co-workers, ${ }^{14,15}$ who in 1999 synthesized a hydrophilic macromolecular network through the formation of carbamate bonds between polyethylenimine (PEI) and imidazole-modified poly(ethylene glycol) (PEG) for oligonucleotide delivery in the cell environment. However, the main purpose of polymeric self-assembly at the nanoscale was also investigated by Akiyoshi's group, ${ }^{16}$ who in 1993 developed the first nanogel via physical cross-linking between amphiphilic polymers.

The nanogel chemical cross-linking approach gives rise to a three-dimensional structure in which bond and connection points are strong and rigid and their mutual distance and distribution define the porosity and the mesh nanosize, representative of the space between macromolecular chains available for drugs or biological fluids to diffuse. ${ }^{17-19}$ On the other side, nanogel engineering through physical cross-linking tends to give more fragile and more easily degradable structures than the covalently cross-linked counterparts; this is due to the weak nature of the polymer connections formed by hydrogenbonding, electrostatic, van der Waals, or hydrophobic interactions, which then make the nanostructure more sensitive to the sol-gel transition caused by environmental stimuli changes. ${ }^{20-22}$ The reduced size is the first highly attractive property of nanogels because they can reach targeted intracellular sites or generate specific intramolecular interactions not easily accessible by macroscopic biomaterials. ${ }^{23-25}$ Generally, researchers have defined as acceptable nanogel dimensions the wide range of $10-1000 \mathrm{~nm},{ }^{8,26}$ while others have registered an optimal size up to $200 \mathrm{~nm}$ in particular for tissue engineering applications. $^{27,28}$ The nanoscale also allows a larger specific surface area, which enhances the stability and bioavailability of the loaded drugs and proteins and increases the response to the physiological environment ${ }^{18,29,30}$ and its variations, such as $\mathrm{pH}$ or temperature. ${ }^{31-33}$ According to this point, their surface area can be used for postfunctionalization to control the nanogel pathway toward a specific target and the cargo release kinetics. $^{34-36}$ On top of that, nanogels present a distinctive feature related to the ability to swell by incorporating a great amount of water or biological fluids while maintaining their structural integrity; in this way, nanogels introduce swelling behavior, which is characteristic of macroscopic hydrogel systems, ${ }^{37-40}$ to the nanoscale. Their physical behavior is 
strongly influenced by the ability to swell in contact with compatible solvents: ${ }^{41-43}$ the contact between nanogels and the solvent molecules leads the latter to attack the polymeric surface and penetrate into the network. Usually, the polymer entanglement starts expanding, allowing other solvent particles to come up to the core of the system. Referring to the nature of these biomaterials, the polar hydrophilic groups are the first to be hydrated upon contact with an aqueous polar solvent, giving rise to the formation of the primary bound water.

The progressive swelling results in an elongated configuration of the polymer chains between the junctions, counterbalanced by an elastic retroactive force that prevents the deformation of the system. The resulting stretching-shrinking equilibrium allows nanogels to be characterized by a core formed by $90-95 \%$ $\mathrm{w} / \mathrm{w}$ aqueous solution and makes them different from micelles and other polymeric nanoparticles used as delivery systems, such as dendrimers and liposomes. ${ }^{44-47}$ Moreover, nanogels can be swelling-responsive according to the use of thermodynamically good solvents, $\mathrm{pH}$, ionic strength, and temperature variation: nonfavorable conditions can promote deswelling behavior and affect the size. ${ }^{18,42}$ Furthermore, the ability to retain water allows for the exchange of ions and metabolites with tissue fluids to maintain the biological chemical balance with the surrounding environment and the diffusion of biomolecules, making them generally biocompatible. ${ }^{48-50}$ Since nanogels have the features of both nanoparticles and hydrogels mentioned above, they can be categorized as soft materials, which combine the properties of solids and fluids. This physical nature influences the surface tension, the biodistribution, and the circulation time in the targeted cells or organs because the resulting intrinsic deformability permits them to cross physiological barriers (such as spleen, liver and lung tissues) more easily than other stiffer nanoparticles, which would be trapped during the biological pathways. Indeed, the circulation half-life is longer as discussed by Zhang and co-workers ${ }^{51}$ and by Hendrickson and colleagues, ${ }^{52,53}$ who investigated the possibility of applying limited pressure to drive nanogels into pores smaller than their dimensions without affecting their structural integrity. Fundamentally, these aspects identify nanogels as a novel generation of delivery systems thanks to the ability to tune their final configuration according to the biological needs: structural uniformity, large surface area, charge, stability, stimuli responsiveness, minimal toxicity, and high encapsulation capacity are the pivotal parameters for the synthesis of functional tools for intracellular and selective drug or gene delivery.

\section{NANOGEL FORMATION}

Nanogels are synthesized using both natural and synthetic polymers. As widely reported in the literature, the former ones are generally protein polymers, involving collagen, albumin, and fibrin, and polysaccharide polymers, such as chitosan, hyaluronic acid, chondroitin sulfate, agarose, alginate, cellulose, and heparin, whereas the synthetic polymers are poly(lactic acid), PEI, PEG, polyglycolic derivatives, polyacrylates, polymethacrylates, and poly( $\varepsilon$-caprolactone $)$. They are characterized by the presence of specific chemical groups that are functional for the formation of covalent links or physical interconnections to design the three-dimensional network, including hydroxyl, amide, carboxamide, thiol, and sulfo groups. These same residual groups, arranged along the macromolecular chains, are mainly responsible for the capacity of the nanogel to absorb water. $^{54}$ As previously introduced, nanogel synthesis can be performed through chemical reaction, which concerns the heterogeneous polymerization of low-molecular-weight monomers or the cross-linking of polymer precursors. ${ }^{18,53}$ The first strategy utilizes controlled/living radical polymerization with specific initiators or macroinitiators to prepare nanotools with different composition, dimensions, and architecture and to incorporate functional groups inside the network or on the surface, which in the end facilitate the multivalent bioconjugation. ${ }^{56-59}$ On the other hand, nanogels obtained by linking of polymer precursors are generally made of self-assembling amphiphilic or triblock copolymers or composed of polymers having different types of reactive sites that can be directly used in the formation of covalent bonds. This chemical cross-linking strategy includes a wide range of experimental pathways based on the principles of click chemistry, ${ }^{60,61}$ thiol-disulfide exchange, ${ }^{62-64}$ Schiff base reaction, ${ }^{65,66}$ photo- or thermally induced cross-linking, ${ }^{67,68}$ amide bond formation, ${ }^{69,70}$ enzymemediated cross-linking, ${ }^{71,72}$ catalyzed coupling, ${ }^{73}$ and the chemistry of ketones, aldehydes, epoxides, or other groups. ${ }^{74}$ Moreover, they can be used for the preparation of core-shell structures or micelles by tuning of the spatial organization of the molecules and the consequent interaction with external biomolecules. $^{75-77}$

Considering the features of the materials used, nanogel formation could be addressed to physical cross-linking; the synthesis occurs under mild conditions, and the driving forces to form the nanostructure are strongly correlated to the specific nature of the polymers and include electrostatic, host-guest, and hydrophobic/hydrophilic interactions..$^{20,78,79}$ In particular, the last of these are generally due to the polymer chemical structure that presents a hydrophilic framework and several grafted hydrophobic moieties. As result, association of the same pattern groups provides a large number of cross-linking points, creating the nanogel. Examples are related to the use of cholesterol and polysaccharides ${ }^{34,80}$ or pullulan. ${ }^{81,82}$ In addition to the discussed chemical and physical approaches, an innovative trend is the fabrication of polymeric nanosystems using particle replication in nonwetting templates (PRINT) technology. This is a sophisticated method to obtain monodisperse particles from a wide range of matrix materials, including biocompatible and biodegradable polymers. ${ }^{47}$ PRINT uses nonwetting elastomeric molds of fluoropolymers that enable high-resolution imprint lithography and eliminate the formation of an interconnecting film between the molded objects. Here, particles can be designed through precise control over the size (from a few nanometers to several micrmeters), shape, composition, surface properties, physicochemical properties, and cargo (intended as hydrophilic or hydrophobic drugs, biological compounds, proteins or peptides, contrast agents, or fluorophores). ${ }^{83}$ The result is significant tunability of the produced biotools according to the application-related and environmental stimuli. ${ }^{84,85}$ Another recent method that has received great attention is molecular imprinting. ${ }^{86,87}$ By means of this strategy, nanogels can be used as protein receptors through electrostatic interactions and can then be developed as sensors. All of these domains offer options to meet the specific parameters for each application. In particular, the size distribution and the stability in dispersion solutions represent fundamental nanogel constraints.

The nanoscale criteria are addressed through two approaches. The first one considers the nanogel formation from a "bulk matrix", such as macroparticles, clusters, or polymeric sol-gel systems. However, the nanogel surface could present imperfections and a nonhomogeneous component distribution 

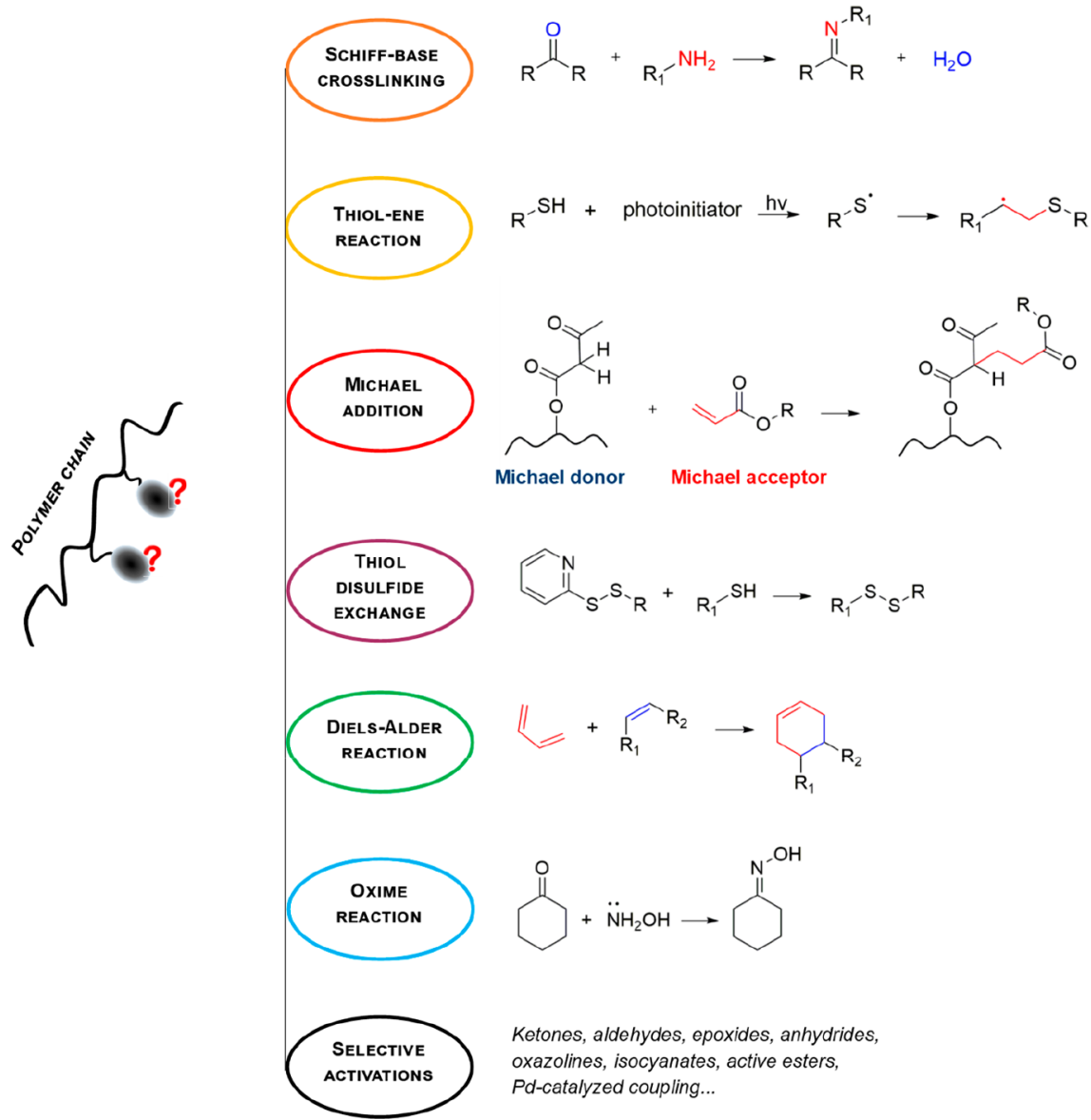

Figure 2. Scheme of the main polymer functionalization techniques.

in the core or at the surface. ${ }^{88}$ The second one is based on molecular design, starting from molecules or clusters that are interconnected via chemical or physical reactions. In this context, the chemical linkage of monomers, polymers, and copolymers represents a convenient technique to assemble shellcross-linked nanogels. ${ }^{55}$ Furthermore, the nanogel stability can be modulated according to the size, nature, and chemical composition of the polymer network. The synthesis of responsive nanogels is widely recognized, and in these studies, the search for temporary and sensitive stability is pivotal: lightresponsive, temperature-responsive, $\mathrm{pH}$-responsive, and redoxresponsive polymers are used to perform selective reception of chemical and biochemical signals based on physical interactions or chemical reactions between functional groups in the polymer and signaling biomolecules. ${ }^{88,89}$

\section{POLYMER FUNCTIONALIZATION}

The study of physical and chemical properties of polymers involved in nanogel synthesis has led to the development of different cross-linking techniques. In particular, the presence of characteristic groups encourages the use of a polymer in some molecule chemistries but limits its application in other aims. The choice of naturally derived materials can be justified by their ability to provide structures extremely comparable to living tissues or stimulating cellular responses, supported by high biocompatibility in vitro and in vivo. Otherwise, the use of synthetic polymers allows setting of the mechanical properties, degradation, and composition of the final nanosystem. The smart combination of them is the first approach to overcome the reciprocal drawbacks and create a versatile carbon-based system representing the closest approximation to biological tissues and materials that is suitable for drug and gene delivery. ${ }^{90}$ Postpolymerization modification offers routes to perform specific grafting of functional groups to the long side chains, macromolecule coupling within the short term, and elaboration of nanoarchitectures to attain the desired mass transport properties, size, porosity, and sensitive interactions with the biological environment.

The generation of a reactive modified polymer substrate represents a preliminary step to promote the reaction between the functional groups along the polymer chain and the other chemical entities supporting drug encapsulation or grafting of DNA or RNA sequences. The state of the art in the field of grafts over existing polymers involves a wide range of techniques, especially considering the ongoing development of new chemical transformations, as briefly illustrated in Figure 2. Blasco and co-workers ${ }^{91}$ widely discuss polymer functionalizations obtained in the following ways:

- Activation of esters to form amide bonds under mild conditions. Amides are among the most versatile linkages in organic chemistry, and they are characterized by unique stability toward extreme chemical environments. Esters have emerged as some of the most adjustable moieties for polymer functionalization.

- Click chemistry approaches, which ensure the activation of molecules and polymers through the introduction of compatible click functional groups and the successive formation of stable conjugates. Copper-catalyzed or 
copper-free strain-promoted azide-alkyne cycloadditions are modular reactions with simple reaction conditions (including aqueous conditions) that give very high yields and have a high tolerance of functional groups. They are stereospecific and generate inoffensive byproducts that can be removed without the use of chromatographic methods. Functionalization with azide and alkyne groups offers the opportunity to link molecules such as peptides, proteins, carbohydrates, and lipids to other biomolecules or polymer scaffolds to generate biohybrid materials with a diverse selection of improved properties. ${ }^{92-95}$

- Thiol chemistry, including free radical thiol-alkene and thiol-alkyne reactions, nucleophilic Michael addition, disulfide exchange, and thiolactone double modification. The radical- and light-mediated reactivity of thiols with carbon-carbon double bonds combines the advantages of click reactions with the benefits of a photoinitiated process activated at specific times and locations, giving rise to a powerful method for the chemical synthesis of tailorable materials. ${ }^{96}$ Indeed, they are characterized by quantitative yields, high reaction rates, compatibility with environmentally benign solvents, no complex product purification techniques, insensitivity to ambient oxygen or water, and the formation of a single regioselective product. $^{97,98}$ The other way based on electrophile and nucleophile interactions offers polymer side-chain and end-group modification using mild reaction conditions, high functional group tolerance, a large host design of branched, linear, or network polymers, high conversions, and favorable reaction rates in emerging technologies including biomedical applications such as gene transfection, cell scaffolds, and tissue replacements. ${ }^{99,100}$

- Isocyanate modifications through the addition of alcohols, amines, or thiols. These promote fast reaction kinetics, the stability of isocyanates toward radicals, and high yields of functionalized polymers. ${ }^{101}$ However, the unreserved application of these strategies is severely limited by the toxicity and the instability/sensitivity of isocyanate-containing polymers to the potential degradation effects of moisture. ${ }^{102,103}$

- Imine and oxime linkages, allowing bond reversibility due to the imine equilibrium and potential oxime hydrolysis under aqueous acidic conditions. The polymer functionalization can be performed according to the final modification aim, selecting the primary amine and the carbonyl derivative partner to form a hydrolytically stable (to preserve the functionalization) or unstable (to release the grafted molecule) linkage. ${ }^{104-106}$

- Ring-opening reactions, which represent very versatile methods for polymer transformations. The nucleophilic ring opening of strained heterocycles, such as epoxides, ${ }^{107,108}$ aziridines, ${ }^{109,110}$ and azlactones, ${ }^{11,112}$ enables the introduction of desired heteroatoms on the polymer backbone.

- Multicomponent reactions (MCRs), including isocyanide-based, non-isocyanide-based and organometalliccatalyzed reactions. These define methods to introduce a high degree of functional complexity in a single atom modification step. ${ }^{91,113,114}$

Alongside these chemical-based approaches, non-covalent interactions, including van der Waals, hydrogen-bonding, and charge transfer interactions and stereo- and polyelectrolyte complexation, can be exploited to introduce tunable physical modifications on the nanoscale. The self-assembly processes generally involve the use of amphiphilic polymers and therapeutic agents through emulsification-evaporation methods (water/oil or oil/water) and dialysis or ionic polymers and molecules to perform electrostatic interactions. ${ }^{115}$ These polymeric chains are usually characterized by hydrophilic and hydrophobic moieties that are alternatively cross-linked thanks to their radical polymerization synthesis, which allows tuning of the nature, chain length, composition, and pendant groups.

However, the stability of the physically cross-linked nanogels is limited compared with nanosystems produced using polymer chemical functionalization. Polysaccharides, poly(amino acid)s, cholesterol, and chitosan derivatives are commonly used to selfassemble and encapsulate proteins, drugs, or DNA. ${ }^{8}$ The techniques of conjugation between the nanogel polymeric component and the desired functionality need to meet the requirements of an adaptable nanocarrier delivery vehicle. Drug delivery focuses on the tunable loading and release of bioactive molecules as diffusion-controlled, swelling-controlled, or chemically controlled: the functionalization is aimed to introduce chemical moieties for the formation of physical or chemical bonds not only with the cargo but also according to external stimuli such as temperature, $\mathrm{pH}$, pressure, ions, and specific molecular recognition. ${ }^{23,116,117}$ The therapeutic use of proteins, peptides, enzymes, oligonucleotides, RNA, and DNA requires the preservation of the corresponding structures and the gene functions; for these reasons, gene delivery involves functionalization of the nanogel with specific chemical groups or targeting biomolecules that are able to form complexes as lipoplexes and polyplexes and ensure cellular internalization via endocytosis. 118,119

In particular, in vivo nanogel applications demand tailorable circulation half-lives of the therapeutic cargo. The different administration routes, such as oral, intradermal, intraocular, and intravenous, involve transitions through biological barriers, where nanogels are required to prevent quick degradation or metabolism. Drugs and genes are difficult to deliver to specific organs or pathological areas because of clearance and nonspecific uptake in the vascular and reticuloendothelial systems and excretory organs, including kidneys and lungs. One key crossover is the mononuclear phagocytic system, which promotes nanogel accumulation in the liver and spleen, reducing the availability in other organs by the circulating blood. Generally, the nanogel design is performed to limit migration through the normal endothelial channels and guarantee compliance to the defects of solid tumors or inflamed tissues characterized by the enhanced permeability and retention (EPR) effect, for example, by tuning of the nanosystem dimensions, softness, and deformability via orthogonal chemical approaches.

Moreover, grafting of ligands to the polymeric nanostructures allows binding to specific receptors or molecules overexpressed in damaged cells or tissues, improving their retention at the targeted site and promoting their cellular internalization. ${ }^{120}$ The latter can occur following different endocytic mechanisms depending on the nanogel physicochemical properties, such as size, shape, charge, and surface chemical groups. Nanosystem uptake is a complex process that is still under investigation, but a common pathway is generally recognized: endocytosis leads the nanogel to the intracellular vesicles, and then they are addressed to an endosomal or lysosomal scenario. ${ }^{18,121}$ As the nanogel moves toward different biological compartments, it encounters 
$\mathrm{pH}$ variations, enzymatic degradation, or a redox environment, and these are often exploited as standard stimuli to release the nanogel cargo. In particular, gene delivery needs the nanogels to release their entrapped active molecules in the cytosol, where the therapeutic effect is desired, avoiding the degradating action of endosomes and lysosomes. An overview of the recent progress in functionalized nanogels designed for drug and gene applications is proposed and discussed in the following sections.

4.1. Nanogel Design for Drug Delivery. The need to improve classic drug administration routes like oral, intravenous, and intra-arterial was, and still is, one of the main goals of researchers on drug delivery systems. ${ }^{122,123}$ Indeed, while pills and injections have enabled significant medical advances, they are inadequate for the delivery of drugs with short half-lives, poor permeability into cell membranes, and serious toxicity when delivered systemically in high doses. In particular, the main improvement would be the possibility to maintain the drug level in plasma at an effective level for a sustained period of time, avoiding under- and overdosing. ${ }^{124,125}$ In this framework, nanogels appear as a promising tool: they provide large specific surface area, which guarantees the interaction with physiological compartments and enhances the stability and bioavailability of the loaded drugs and proteins. ${ }^{126-128}$

Despite the good results obtained in many applications, such as glucose-sensitive release systems, ${ }^{129}$ several critical aspects are related to the fact that drug release is mostly driven by a pure diffusion mechanism that is very quick because of the high clearance observed in vivo. The hydrophilic nature of encapsulated drugs and biomolecules is not enough to control the release mechanisms, and therefore, the necessity to develop nanogels that are able to delay release rates or allow multiple release kinetics, different from pure Fickian ones, is very demanding. ${ }^{130,131}$ In order to overcome these drawbacks and to attenuate diffusional release of biomolecules, several research groups ${ }^{132-134}$ introduced biorthogonal strategies to create an affinity bond between drug molecules and the polymeric network, covalently linking a small protein receptor module to gel systems. Similar procedures were also followed by Ossipov and co-workers, ${ }^{135}$ who used a carbazone coupling involving aldehyde groups to attach hyaluronic acid (HA) macromolecules to the neutral poly(vinyl alcohol)-doxorubicin prodrug system (Figure 3). Simultaneously, the carbazone coupling was accompanied by a thiol-disulfide exchange reaction that transformed the forming macromolecular conjugate into self-associating nanostructures able to decrease the drug release rates. In these cases, drug release is not driven by Fickian diffusion but delayed by the stability or affinity of the bond between the drug and polymer, with higher stability leading to slower release kinetics. These drug delivery vehicles are strongly internalized in the cytosol of cells overexpressing the CD44 receptor, promoting the drug cure in breast cancer. This approach guarantees the controlled and sustained release of drugs/biomolecules on one hand, but on the other hand, the chemical modification of the active principals may change their efficacy. ${ }^{132,136}$ Polymer functionalization could also be used to enhance non-covalent interactions between drug molecules and polymer moieties. In this direction, Le and co-workers ${ }^{137}$ used furfuryl groups in the poly(styrene-alt-maleic anhydride) block to incorporate a redox-responsive linkage, and the carboxylic acid moieties generated through functionalization acted as a $\mathrm{pH}$ responsive part. Doxorubicin, a model anticancer drug, was loaded into the core of the nanogels primarily by ionic
A

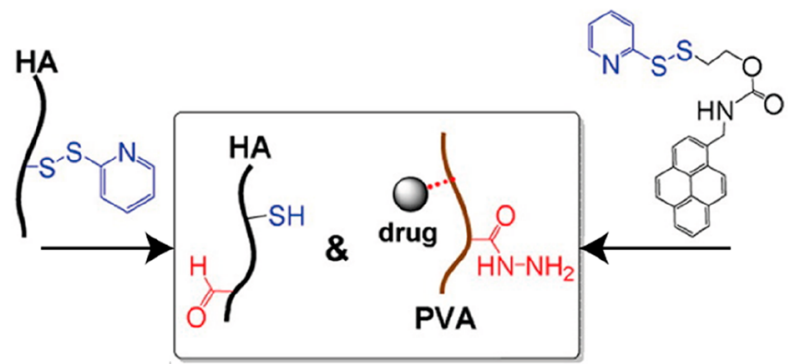

B

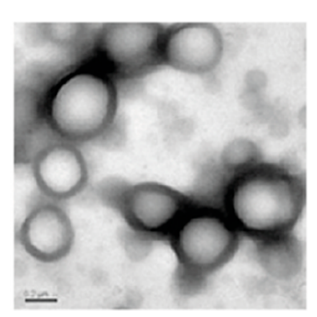

C

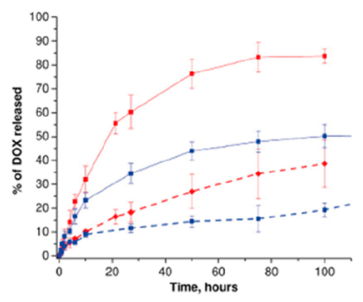

Figure 3. (A) Thiol-disulfide exchange reaction and carbazone chemistry employed to construct a disulfide nanogel of hyaluronic acid (HA) macromolecules with the carbazone-linked poly(vinyl alcohol) prodrug of doxorubicin (PVA-DOX). (B) TEM image of the synthesized nanogels (scale bar $=200 \mathrm{~nm}$ ). (C) Profiles of drug release from the prodrug (red curves) and from the nanogels (blue curves) at pH 5 (solid lines) and pH 7.2 (dashed lines). Adapted from ref 135. Copyright 2013 American Chemical Society.

interactions with carboxylates of the core blocks, and a highest drug loading capacity was obtained.

Polymer functionalization applied to nanogels could also be used to direct drug delivery within specific cells. ${ }^{120,138-140} \mathrm{Su}$ and co-workers ${ }^{141}$ developed fluorescent bovine serum albumin (BSA)-encapsulated gold nanoclusters (AuNCs) conjugated onto the nanogel surface, followed by functionalization of the tumor-targeting peptide iRGD onto the BSA for tumor targeting. The iRGD motif mediated specific targeting to tumor and endothelial cells and enhanced cellular uptake of the nanostructures, allowing targeted release of doxorubicin. Chambre et al. ${ }^{116}$ considered copolymers containing the thiolreactive maleimide group as good strategy to install $\mathrm{N}$ hydroxysuccinimide groups containing carbonates that enable conjugation of amine-containing drugs through acid-labile carbamate linkages. Drug delivery systems inspired by natural particulates hold great promise for targeted cancer therapy. In this direction, Yu et al. ${ }^{142}$ verified that an endosome formed by internalization of a plasma membrane has a massive amount of membrane proteins and receptors on the surface, which are able to specifically target homotypic cells loaded onto nanogels. Among the nanostructure modifications to tune cell uptake or blood circulation, PEGylation represents one of the most commonly used, ${ }^{138,143-145}$ and it is well-known in both the scientific community and industrial practice. ${ }^{146}$ PEG grafting turns out to be suitable to tune the loading and delivery of drugs from nanogels. ${ }^{147-149}$

Furthermore, the use of HA-based nanogels represents an alternative for the treatment of cancer cells because of the CD44 and RHAMM receptors, which are overexpressed in various types of tumors. ${ }^{150,151}$ The functionalization of $\mathrm{HA}$ with epigallocatechin-3-gallate (EGCG) via a condensation reaction and the introduction of a PEI moiety allowed to Liang and coworkers to synthesize intracellular nanogels for targeted cancer 


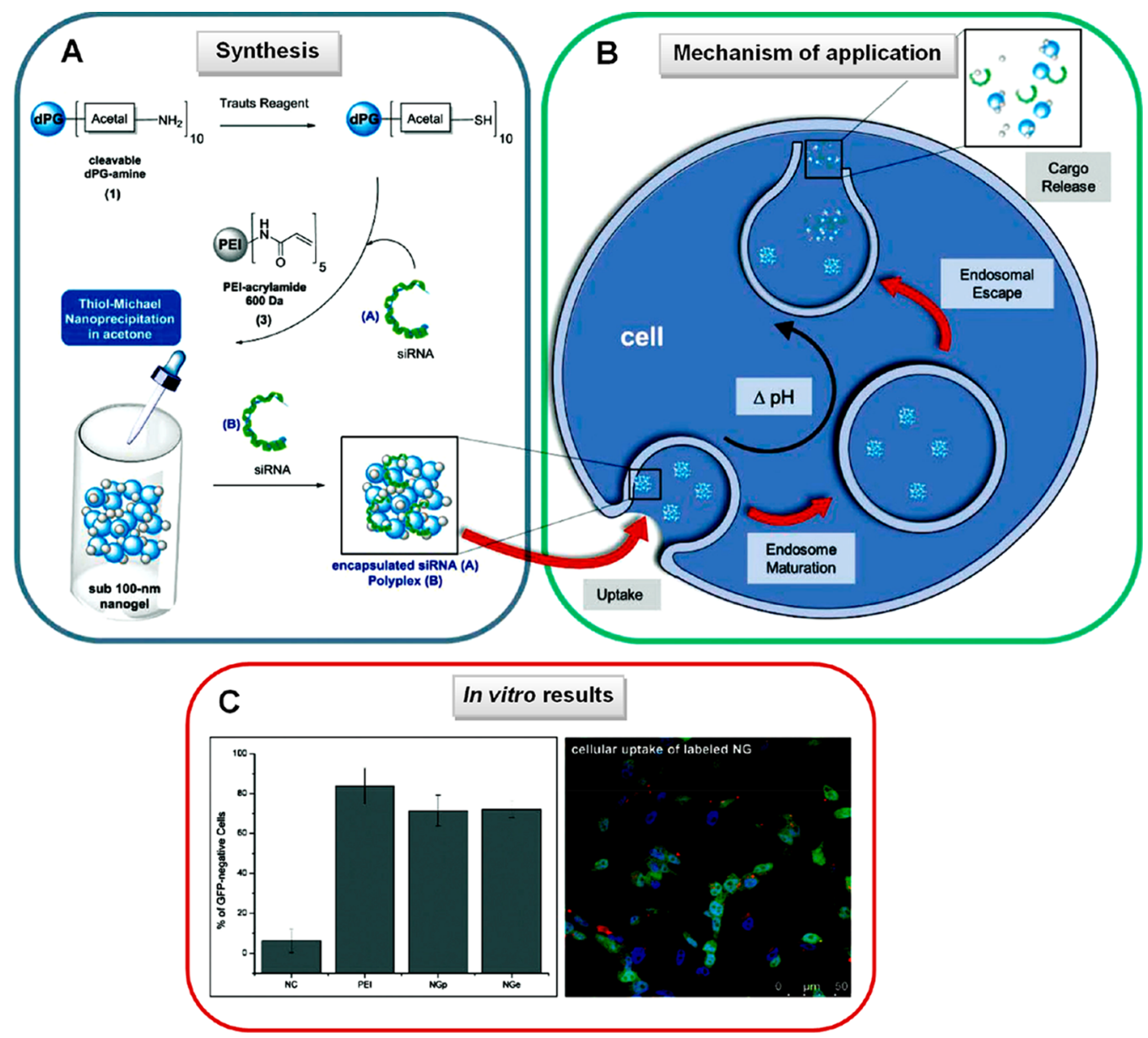

Figure 4. (A) Synthesis of $\mathrm{pH}$-sensitive nanogels using the dPG and PEI functionalization approach. (B) Mechanism of gene delivery. (C) In vitro results of transfection efficacy: comparison among untreated control cells (NC), PEI, and nanogels functionalized with encapsulated (NGe) or complexed genetic material (NGp). The GFP-siRNA expression is higher in cells treated with nanogels than in the control and comparable to the use of only PEI, which is potentially toxic. Adapted with permission from ref 157 . Copyright 2017 Royal Society of Chemistry.

therapy. ${ }^{152}$ An innovative approach regards the use of enzymatic polymerization to synthesize cationic nanogels that can be biodegraded in situ by an endogenous enzyme and release gene materials without any external stimuli.

4.2. Nanogel Design for Gene Delivery. Gene therapy regards the administration of foreign genetic materials (DNA or RNA) into the human body to cure diseases. ${ }^{153}$ The potential of this approach is strictly dependent on two essential constituents: the design of an effective nucleic acid sequence that can be expressed or used for silencing at a target site, correcting genetic defects or inhibiting the synthesis of malignant proteins, ${ }^{154}$ and the development of an efficient and safe delivery method to transport the genetic sequence to the specific treatment area or organ.

Nowadays, antisense oligonucleotides, plasmid DNA (pDNA), small interfering RNAs (siRNAs), and micro RNAs (miRNAs) are involved in promising gene therapies to diagnose and counteract numerous diseases such as cancer, neurodegenerative disorders, and viral infections through the selective inhibition of mRNA sequences. ${ }^{8}$ However, the main limitations are related to the easy inactivation and premature degradation of the genetic cargo during intracellular delivery. Nanogel functionalization leads to the design of gene delivery systems that are able to cross the cell membrane, preserving the therapeutic load from endogenous enzymes, to reach specific cells through bioconjugated molecular recognition and finally to provide controlled release of the genetic material in the perinuclear or nuclear area without inducing cytotoxicity and immune response. In the last years, researchers have shown that modified nanogels ensure high transfection efficiency, high cellular stability, low toxicity, and low immunogenicity as nonviral carriers. ${ }^{155,156}$ Dimde and co-workers ${ }^{157}$ developed the double functionalization of dendritic polyglycerol (dPG) with $\mathrm{pH}$-sensitive benzacetal bonds and terminal amine groups and coupled it with PEI-modified acrylamide through a thiolMichael reaction. The resulting cationic nanogels fulfilled the dimensional criterion (sub-100 $\mathrm{nm}$ range) for the most efficient siRNA delivery in targeted HeLa cells, and the presence of acetal linkers in the nanogel network enabled controlled intracellular release of the siRNA cargo, resulting in improved silencing in green fluorescent protein (GFP)-expressing cells. Moreover, the dPG-PEI conjugation solved the adverse effects of PEI toxicity thanks to the $\mathrm{pH}$-degradable nanogel architecture, preserving the high transfection efficacy, as shown in Figure 4. The nanogel traceability in in vitro studies was performed using rhodamine isothiocyanate, highlighting the versatility of dPG functionalization in this gene delivery system.

A wide range of studies have focused on the use of poly(amido amine)s to address gene therapy needs. ${ }^{158}$ These peptidomimetic polymers can be rapidly synthesized from amine derivatives and bis(acrylamide)s through Michael-type addition reactions, and the polymerization allows the introduction of 


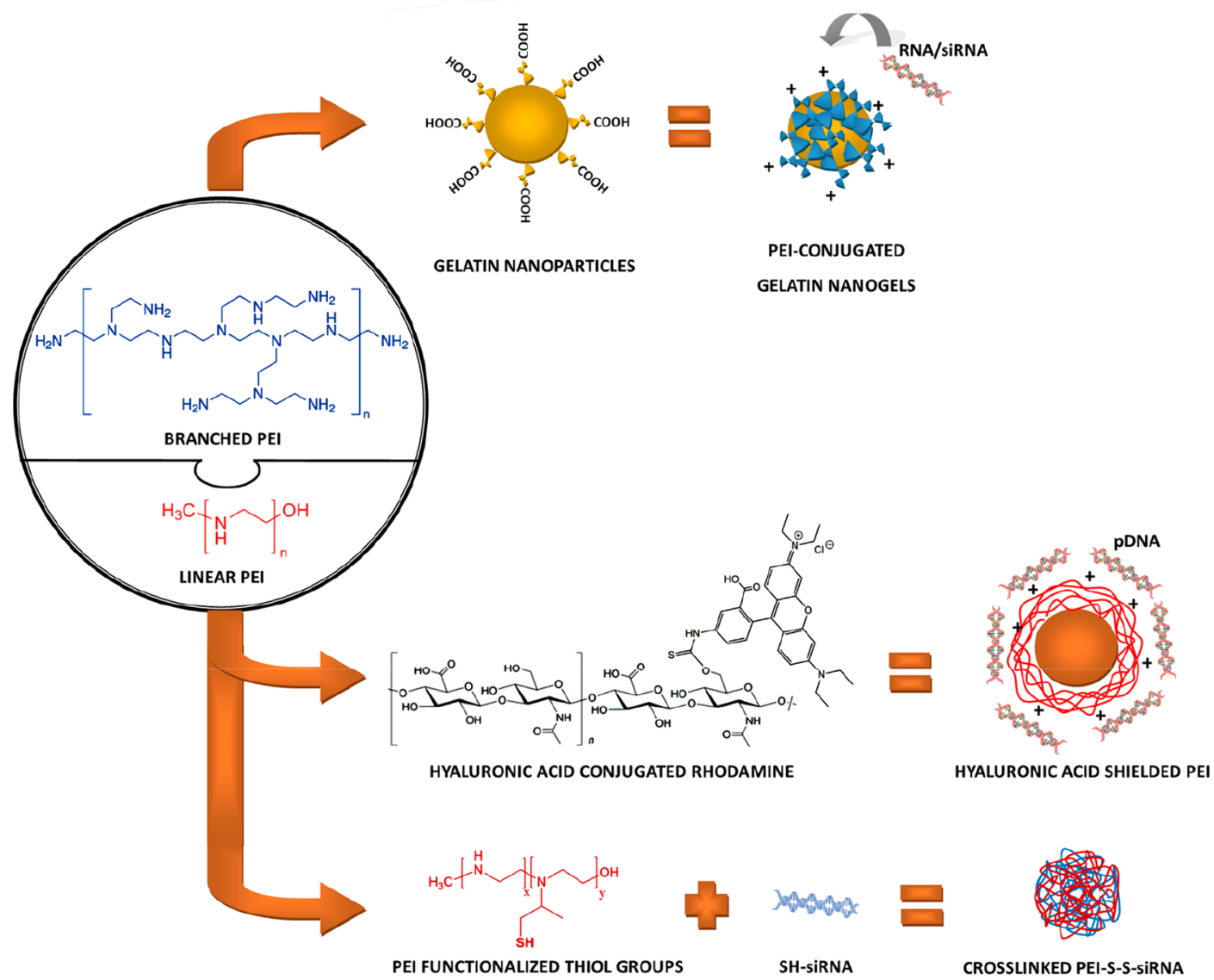

Figure 5. Scheme of PEI functionalization approaches developed by Mimi, ${ }^{165}$ Park, ${ }^{166} \mathrm{Hong},{ }^{167}$ and their co-workers to design nanogels for gene delivery.

different chemical functionalities in the backbone and side chains. The colloidal stability of nanopolyplexes, gene encapsulation ability, polyplex unpacking, cytotoxicity, and transfection efficiency could be tuned according to the introduced chemical moieties. As discussed by Jones and coworkers, ${ }^{159}$ one leading point is related to the number of available amine groups: polymers with one tertiary amino group in the repeating unit structure (such as the product of the reaction between methylenebis(acrylamide) and monomethylamine) was characterized by a lower capacity to bind DNA than polymers with two tertiary amino moieties (for example, using methylenebis(acrylamide) and dimethylethylenediamine), but their substitution with heterocyclic amines, such as piperazine, prevented the polymer from efficient DNA binding because of the ring steric hindrance, which did not allow the DNA to approach closely. Moreover, the different structures of grafted amine moieties affected the spatial rearrangement of the final nanostructure, the size, the stability, and the amount of DNA cargo that could be released. Another functionalization regards carboxylic acid groups to ensure the escape of the nanocarrier from the endosomes into the cytosol for the final gene delivery into the nucleus without enzymatic degradation. In the work by Griffiths et al., ${ }^{160}$ amphoteric poly(amido amine)s with pendant carboxyl moieties showed $\mathrm{pH}$-sensitive conformational changes due to protonation/deprotonation of the $-\mathrm{COOH}$ and $-\mathrm{NH}_{2}$ groups, similar to the behavior of fusogenic peptides, which were able to disrupt the endosomal membrane, permitting the nanopolyplex to escape from endosomes; the carboxylic acid functionalization could be exploited to preserve the gene cargo from enzymes present in the acidic endosomes and liposomes. ${ }^{161}$ In addition, an improvement in the transfection efficiency can be obtained through the synthesis of disulfidebased poly(amino amine) $\mathrm{s}^{162}$ according to the cleavability of the $S-S$ linkage in the presence of intracellular glutathione or cysteine.

In addition to these pivotal functionalizations, the introduction of multiple pendant groups in the poly(amino amine)s, such as hydroxyl moieties, heterocycles, methyl groups, and nitrogen, leads to the design of enhanced and tailored nanostructures as discussed by Lin and Engbersen. ${ }^{158}$ Not only the ionic interactions, which primarily depend on the charge density of the polymers, but also structural flexibility affect the design of efficient gene delivery nanosystems. Among the polymers used in nanogel synthesis, PEI represents a reference in gene therapy because of its positive charge, which can compact the nucleic acids into the nanocomplex and supports the cell membrane crossing via the endocytotic pathway and endosomal escape effect, exhibiting high transfection efficiency. However, the use of only PEI is greatly restricted by its cytotoxicity, and it also increases the nanocomplex dimensions to beyond the optimal range for clinical siRNA and DNA delivery. ${ }^{163}$ PEI is characterized by a branched or linear configuration. The former has higher chemical reactivity and can form smaller complexes with DNA under salt-containing conditions, whereas the linear chains are generally less toxic and have a higher potential of transfection. ${ }^{164}$ For these reasons, alternative PEI functionalizations have been proposed. Mimi and co-workers ${ }^{165}$ synthesized nanogels made of a biodegradable gelatin core and a shell of 
conjugated branched PEI. The gelatin core was formed through the thermal cross-linking reaction between the amino and carboxylic groups of the gelatin molecules, and the PEI covalent grafting was obtained through coupling between the residual gelatin carboxylic acid and polymer primary amine groups in the presence of $N$-ethyl- $N^{\prime}$-(3-(dimethylamino)propyl)carbodiimide hydrochloride (EDC). The nanogels resulted in a 4-fold reduction in toxicity compared with PEI-based nanogels, and they were able to entrap, protect from enzymatic degradation, and deliver siRNA to HeLa cells. The gene silencing effect was estimated to be higher (up to 70\%, using ASS 1 as the target gene) than that of the commercially available transfection agent Lipofectamine (about 32\%). Park and his team $^{166}$ proposed another PEI functionalization using HA instead of gelatin that allowed the synthesis of HA-shielded PEI nanogels loaded with pDNA.

In this case, the polymer linkage and electrostatic interactions gave rise to a system that could be internalized by human mesenchymal stem cells and HeLa cells exploiting the selective receptors located in the plasma membrane. These nanogels could be useful to perform stem cell internalization and induce in situ differentiation. The functionalization of PEI with thiol groups represents another good alternative to synthesize crosslinked nanogels carrying thiol-terminated siRNA, as proposed by Hong and co-workers. ${ }^{167}$ This approach allows the design of inter- and intramolecular networks with reductively cleavable bonds in the cytoplasm environment, releasing the biologically active siRNA; it was demonstrated that these nanogels exhibited significantly enhanced cellular uptake and gene silencing efficiency compared with the corresponding PEI nanogels without the thiol cross-linking. A representation of the main PEI modifications discussed is shown in Figure 5.

Other nanogel modifications have been reported, such as the introduction of a PEG layer exploiting the residual amine groups of the standard nanogels. This, for example, is discussed by Tamura and co-workers, who studied the delivery of siRNA from nanogels composed of a polyamine cross-linked core coated with PEG chains. ${ }^{168}$ The promising role of the combination ethyleneamine groups was also tested by $\mathrm{Li}$ and co-workers, who functionalized poly(glycidyl methacrylate) (PGMA) with ethylenediamine. ${ }^{169}$ This configuration allowed grafting of the antioxidant lipoic acid, producing cationic reducible nanogels characterized by plentiful disulfide bonds. As a result, the modified nanogels exhibited efficient performance of pDNA transfection and could transport MALAT 1 siRNA into hepatoma cells, suppressing the cancer cell proliferation and migration.

4.2.1. Nanogels for Nucleic Acid Delivery. Effective highly controlled conjugation methods have been developed to deal with the delivery of nucleic acids to the central nervous system: nonviral nanomaterials have been the potential solution in transport of active molecules (drugs, DNA, and RNAs) across the blood-brain barrier (BBB) thanks to their flexible design and easily tailored properties compared with the viral vectors and the involved risks of induced immune responses and unwanted mutagenesis. ${ }^{170,171}$ In this field, nanogel functionalizations are generally aimed at loading either complex genes or siRNA for the treatment of neurological diseases or fluorescent markers for nanocarrier trafficking or brain imaging. ${ }^{172,173}$ The design of multifunctional nanogels involves not only their surface modification with PEG to improve the biocompatibility and to enhance the blood circulation time but also the use of antibodies or homing peptides with high brain affinity to cross the $\mathrm{BBB}$ and maintain the cargo within the neurological environment. ${ }^{173}$ The selective targeting requires the use of ligands that specifically interact with receptors expressed on the cerebral microvascular endothelial cell surfaces, resulting in nanogel binding and internalization into the brain parenchyma.

The use of cationic materials, such as PEI and poly(L-lysine), amino or ammonio group-based copolymers containing acrylates, methacrylates, or radially branched poly(amidoamine) (PAMAM), is also becoming popular. For example, Hwang and co-workers ${ }^{174}$ designed a PEI polyplex functionalized with rabies virus glycoprotein (RVG) to encapsulate and deliver miRNA in the brain. The conjugation approach involved the modification of PEI amine groups at thiol groups to form disulfide-cross-linked dimers, and RVG peptide was linked to PEI chains through a functionalized spacer composed of PEG with $\mathrm{N}$-hydroxysuccinimide (NHS) and maleimide terminal moieties (NHS-PEG-MAL). The addition of anionic miRNA to the resulting polycationic polymer gave rise to the formation of nanosized particles by electrostatic interactions. In this way, the orthogonal chemistry allowed the synthesis of selective nanogels that can cross the BBB, be specifically taken up by neuronal cells expressing the acetylcholine receptor (the effect of the RVG motif) with no cytotoxic consequences on cell populations (the effect of the PEI-PEG combination), and finally also provide tailored miRNA release in situ in the brain parenchyma without loss of the therapeutic cargo in other regions. Also, cross-linking between PEI and PEG was an efficient approach for intracellular release. Vinogradov and co-workers proposed different techniques: functionalization of nanogels with biotin $p$-nitrophenyl ester, reaction with PEI amino groups, and further conjugation with transferrin and insulin; ${ }^{173}$ modification of the PEI nanogel component with folic acid to ensure the formation of complexes with nucleoside 5 'triphosphates in aqueous media; ${ }^{176}$ and modification of the nanogel with Pluronic. ${ }^{177}$ Dissolved in water, these polymeric networks were able to immobilize negatively charged bioactive compounds, such as oligonucleotides, siRNA, DNA, and proteins, through nanogelcargo ionic interactions. In particular, the oligonucleotides incorporated in nanogels crossed an in vitro BBB model, and their in situ degradation decreased as a result of the protection effect of the nanoscaffold. This meant increased uptake of the carried oligonucleotides into the neural target cells and reduced unwanted and delocalized uptake in the liver and spleen.

Further functionalizations were proposed using other polymeric matrices, such as chitosan, poly(acrylic acid), Pluronics, alginate, and polyacrylamides, that were linked to peptides or antibodies with the common aim to ensure a targeted interaction with receptor-mediated endocytosis, to provide stability, and also to avoid premature endosomal escape close to the cell membrane, which would have led to a consequently longer distance to reach the nucleus, increasing the hazard of degradation. ${ }^{178,179}$

4.2.2. Nanogels for Codelivery of Genes and Growth Factors. Gene therapy draws its efficacy also from the codelivery of specific genes and growth factors, in particular in the context of cell differentiation. The encapsulation of growth factors and genes can involve functionalization of nanogels with peptides, polysaccharides, or drugs. For example, Yang and co-workers ${ }^{180}$ reported the synthesis of heparin-conjugated supramolecular Pluronic nanogels with a PEI coating to entrap vascular endothelial growth factor (bFGF) and pDNA. The Pluronic functionalization was obtained through amide linkages and then complexed with PEI. These nanogels were able to be taken up by 


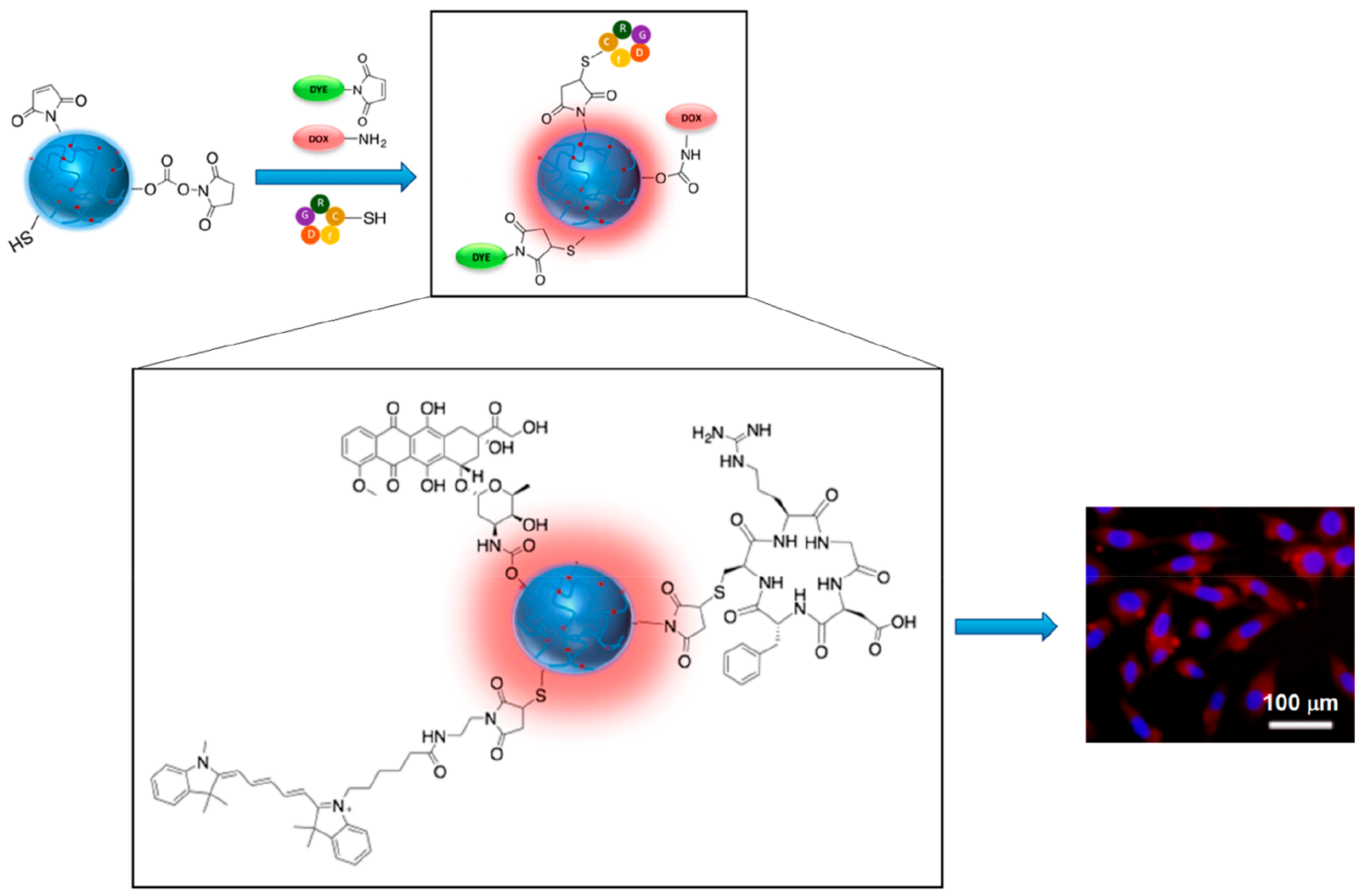

Figure 6. Synthesis of traceable and curative nanogel systems in cell culture: the polymer functionalizations offer the opportunity to link drug (drug delivery) and dye (imaging) to the nanogel, obtaining a multifunctional tool. Adapted from ref 116. Copyright 2018 American Chemical Society.

human endothelial progenitor cells and promoted the endothelial pathway toward the regeneration of vessels in the ischemic limb model system. The PEI coating represents an alternative to the use of a PEG coating, which, as discussed in the literature, ${ }^{181,182}$ can shield the cationic surface of the nanocarrier, decreasing protein/gene absorption and recognition by the phagocytic system and hence limiting the transfection or knockdown efficiency in vitro and in vivo. To overcome these debated aspects, Nishimura and co-workers ${ }^{183}$ prepared maltopentaose-functionalized cholesteryl poly(L-lysine) nanogels and used enzymatic polymerization with glycogen phosphorylase and glycogen branching enzyme to add branched amylose moieties as a nanogel shell. These nanostructures were suitable as siRNA delivery carriers, in particular in murine renal carcinoma, and their cellular internalization was facilitated by the in situ presence of $\alpha$-amylase, which induced hydrolysis of the branched polysaccharide (deshielding), enhancing gene knockdown effects.

Nanogel conjugation with peptides is another approach to perform gene delivery affecting growth factor expression in the treated area. One reference work in this context has been proposed by Blackburn and co-workers, ${ }^{184}$ who synthesized nanogels of methacrylamide and acrylamide and then conjugated the YSA or SCR peptides to the network through maleimide coupling to the cysteine terminal residue of the peptide end. The peptide-labeled nanogels were capable of high siRNA loading and efficient targeting of ovarian carcinomas by receptor-peptide binding; when released, siRNA was available for gene silencing in the cytosol. These nanocarriers also exhibited good protection of siRNA during endosomal uptake and escape thanks to their peculiar osmotic swelling/deswelling behavior in response to the endosomal changes in osmotic pressure and ionic strength occurring during cellular uptake.

4.3. Nanogel Design for in Vitro and in Vivo Tracking. Because of their high degree of stability and good dispersibility, nanogels appear as attractive delivery platforms for theranostic applications: besides their use in drug delivery and gene and protein encapsulation and release, they are also applied in the bioimaging field. ${ }^{116,185}$ The conjugation of fluorescent agents to obtain a polymeric network that is able to meet the requirements of magnetic resonance imaging, in vitro and in vivo tracking, and positron emission tomography demands that nanogels are functionalizable in a tunable way under mild conditions. ${ }^{186}$ In particular, the grafting of the imaging agent needs to be stable and usually uncleavable under a wide range of biological and biomedical environments (e.g., different $\mathrm{pH}$ ). The use of amine, thiol, carbamate, azide, and alkyne groups mainly allows avoidance of premature release of the fluorescent agents and the lability of their resulting bonds. Chambre and co-workers ${ }^{116}$ described well that the thiol-maleimide and carbamate chemistry leads to the design of nanogels with multiple chances of incorporating dyes, and in particular, they used Cy5 maleimide to produce fluorescent traceable nanogels that also carried doxorubicin and peptides (Figure 6).

Cy5 derivatives were generally chosen because of their functional detection in vitro and in vivo without signal overlap with other biological compounds and a lower background.

Cy5-modified azide was proposed in previous work by Mauri and Rossi ${ }^{187}$ to ensure the traceability of the synthesized PEIPEG-based nanogels: PEI was functionalized with an alkyne 
Table 1. Nanogels Used for Drug and Gene Delivery

\begin{tabular}{|c|c|c|}
\hline nanogel type & drugs/genes & technique \\
\hline \multirow[t]{2}{*}{ HA-PVA nanogels ${ }^{135}$} & $\begin{array}{l}\text { doxorubicin } \\
(\mathrm{DOX})\end{array}$ & HA was modified with thiol and aldehyde terminal groups. DOX was grafted on PVA through carbazate bond. \\
\hline & & $\begin{array}{l}\text { Nanogel formation occurred via thiol-disulfide exchange and carbazone coupling. The carbazone cleavability allowed the release } \\
\text { of DOX within cancer cells. }\end{array}$ \\
\hline \multirow[t]{2}{*}{ PEG- $b$-PSMf nanogels ${ }^{137}$} & DOX & $\begin{array}{l}\text { The PEG- } b \text {-PSM copolymer was functionalized with furfurylamine to react with a bismaleimide cross-linker via Diels-Alder } \\
\text { reaction to obtain starlike nanogels. }\end{array}$ \\
\hline & & DOX was loaded into the nanogel, exploiting its ionic interactions with carboxylate groups of the polymer blocks. \\
\hline $\begin{array}{l}\text { poly }(\mathrm{NIPAM}-\mathrm{co}-\mathrm{AA}) \\
\text { nanogel-conjugated } \mathrm{Au} \\
\text { nanoclusters }\end{array}$ & DOX & $\begin{array}{l}\text { Fluorescent BSA encapsulation in the Au nanocluster and conjugation in water to the nanogel structure. The latter was activated } \\
\text { using sulfo-SMCC to link iRGD-SH. DOX was entrapped by steric hindrance. }\end{array}$ \\
\hline PEG-based nanogels ${ }^{116}$ & DOX & $\begin{array}{l}\text { PEG was functionalized with maleimide, thiol, and activated carbonate groups. Nanogels were synthesized via self-assembly using } \\
\text { a dithiol-containing cross-linker. DOX was grafted with a cleavable carbamate bond to perform controlled drug release under } \\
\text { acidic conditions. }\end{array}$ \\
\hline $\begin{array}{l}\text { HA nanogels grafting } \\
\text { endosome membrane } \\
\text { components }\end{array}$ & DOX & $\begin{array}{l}\text { HeLa cells were exposed to } \mathrm{HA} / \mathrm{SiO}_{2} / \mathrm{Fe}_{3} \mathrm{O}_{4} \text { nanoparticles, and } \mathrm{UV} \text { irradiation gave rise to } \mathrm{HA} \text { nanogels via cross-linking } \\
\text { polymerization. }\end{array}$ \\
\hline \multirow{5}{*}{$\begin{array}{l}\text { PEGylated } \\
\text { nanogels }\end{array}$} & DOX, dyes & PEGylation nanogel shell using: \\
\hline & & - PEG-SH on polydopamine \\
\hline & & - PEG-imidazole or PEG-COOH on PEI amine groups \\
\hline & & - PEGMA in ATRP emulsion polymerization of cationic nanoscaffolds \\
\hline & siRNA, pDNA & $\begin{array}{l}\text { Ethylene moieties reacted with amine groups (by polyamine or modified PGMA) to design the nanoscaffold entrapping siRNA or } \\
\text { pDNA. }\end{array}$ \\
\hline dPG-PEI nanogels ${ }^{157}$ & siRNA & Thiol Michael addition between dPG functionalized with benzacetal and amine groups and PEI-modified acrylamide. \\
\hline $\begin{array}{l}\text { poly (amido amine }) \\
\text { nanogels }\end{array}$ & DNA, genes & $\begin{array}{l}\text { Functionalization of poly(amido amine)s with acrylamide, carboxyl, or disulfide groups, respectively, allowed improved DNA } \\
\text { cargo loading and release, protection of genes from enzymatic degradation, and controlled release of genes under glutathione or } \\
\text { cysteine stimuli. }\end{array}$ \\
\hline $\begin{array}{l}\text { branched PEI-based } \\
\text { nanogels }\end{array}$ & RNA & $\begin{array}{l}\text { Covalent linking between PEI and gelatin to reduce the toxicity of PEI, ensure protection of the cargo from enzymatic } \\
\text { degradation, and deliver siRNA to HeLa cells. }\end{array}$ \\
\hline \multirow[t]{2}{*}{$\begin{array}{l}\text { linear PEI-based } \\
\text { nanogels }\end{array}$} & RNA, DNA & $\begin{array}{l}\text { PEI nanogel functionalization with an HA shell to address DNA internalization within human mesenchymal stem cells and HeLa } \\
\text { cells. }\end{array}$ \\
\hline & & As an alternative, thiol moieties could be linked to PEI to deliver siRNA into the cytoplasm. \\
\hline $\begin{array}{l}\text { cationic polymer-based } \\
\text { nanogels }\end{array}$ & nucleic acids & Use of polymers containing amine groups to conjugate peptides or glycoproteins and cross the BBB. \\
\hline $\begin{array}{l}\text { Pluronic- and HA-based } \\
\text { nanogels }\end{array}$ & $\begin{array}{l}\text { growth factors, } \\
\text { genes }\end{array}$ & $\begin{array}{l}\text { Nanogel formation using Pluronic or HA with PEI and further conjugation with growth factors, proteins, or antioxidant molecules } \\
\text { for targeted cancer therapies. }\end{array}$ \\
\hline $\begin{array}{l}\text { methacrylamide/acrylamide } \\
\text { nanogels }\end{array}$ & RNA & Functionalization with specific peptides to tune the cargo release through receptor-peptide binding. \\
\hline $\begin{array}{l}\text { nanogels for in vitro and in } \\
\text { vivo tracking }\end{array}$ & $\begin{array}{l}\text { fluorescent } \\
\text { agents, dyes }\end{array}$ & $\begin{array}{l}\text { Modification of different polymers with amine, thiol, carbamate, azide, or alkyne groups to ensure the conjugation with Cy5, } \\
\text { rhodamine, or quantum dots and the traceability of the nanostructure. }\end{array}$ \\
\hline
\end{tabular}

moiety, and through the copper-catalyzed alkyne-azide cycloaddition (CuAAC) reaction, the formation of a stable triazole bond between the polymeric network and the fluorescent dye was obtained, and it was demonstrated to be uncleavable under physiological conditions and able to induce inflammatory cellular conditions. This click reaction strategy was also used to investigate the effect of dye grafting on the nanogel synthesis: ${ }^{188}$ in this case, rhodamine B (RhB) was chosen as the fluorescent agent and modified with an azide group. The $\mathrm{CuAAC}$ reaction between the resulting $\mathrm{RhB}$ and alkynefunctionalized PEI conducted before nanogel formation led to nanostructures characterized by smaller diameters than ones where $\mathrm{CuAAC}$ was performed after the formation of the polymeric network, and this result affected the cellular internalization mechanism. The design of the nanogel for imaging can be performed using both hydrophilic and hydrophobic dyes according to the different conjugation techniques. Indeed, Aktan and co-workers ${ }^{189}$ showed the functionalization of thiol-maleimide-based nanogels through the conjugation of a thiol-bearing hydrophobic dye (BODIPY$\mathrm{SH}$ ) and $\mathrm{N}$-(fluoresceinyl)maleimide, affording a targeted imaging agent for cellular uptake. Moreover, the additional nanogel functionalization with a cyclic peptide combined with $\mathrm{N}$-(fluoresceinyl)maleimide gave rise to improved cellular internalization due to the selectivity of the peptide, checked thanks to the simultaneous presence of a fluorescent agent in the polymeric scaffold. Another imaging approach regards quantum dots (QDs). They are commonly used as imaging nanocarriers, but their cytotoxicity defines severe limits of application. The functionalization of PEI with QDs is a strategy developed by Park and co-workers ${ }^{190}$ to prepare biocompatible sunflowertype nanogels that are able to carry biomolecules such as nucleic acids and to be internalized by human mesenchymal stem cells with preservation of their viability. Therefore, the introduction of fluorescent agents in nanogel synthesis represents a supporting tool to monitor, test, and verify the efficacy of the produced nanostructure, and it is not a constraint to further functionalizations for the design of an optimal nanogel for drug and gene delivery. ${ }^{191}$

To summarize, Table 1 presents a conclusive list of the types of nanogels discussed and the drugs or genes as well as the strategies of encapsulation used.

\section{CHALLENGES AND NEW PERSPECTIVES FOR FUNCTIONALIZED NANOGELS}

Although progress in the application of nanogels for drug and gene delivery has been dramatic and successful, several main challenges, shown schematically in Figure 7, remain in this field before it reaches clinical applications. First, a better understanding of the mechanisms and pathways behind the intracellular uptake and fate of the nanosystems in complex biological fluids is necessary. Several studies have already been performed 


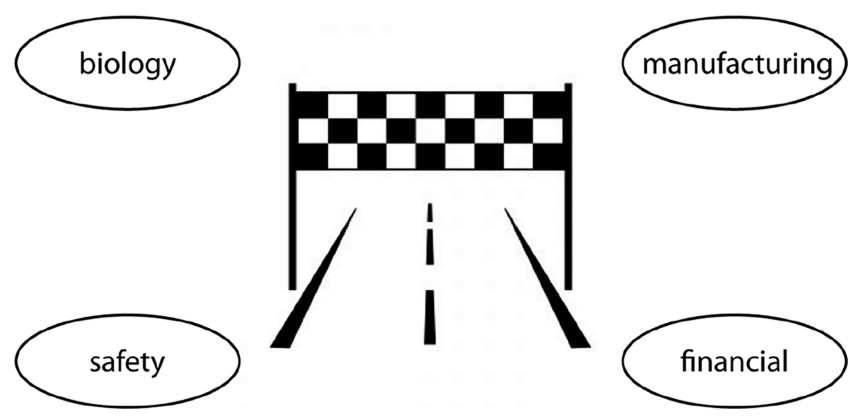

Figure 7. Main remaining challenges in the field of nanogels.

in vivo for neat nanogels, ${ }^{23,192,193}$ and they should be done also for the functionalized ones and then validated in humans. Indeed, current delivery systems could find problems like quick clearance by the immune system and low selectivity and cell targeting, together with difficulty in crossing biological barriers. In this direction, full knowledge of the underlying biology would help to control and tune drug and gene delivery. In parallel, safety concerns should also be taken into high consideration. Nanotoxicity was developed in parallel with nanomedicine with the purpose of studying the potential negative impact of nanoobjects (here nanogels) on biological systems. Preliminary studies revealed that these polymeric networks could contribute to inflammation, damage, and undesired penetration through physiological barriers and come in contact with areas of the body that are particularly susceptible to toxic effects. ${ }^{194,195}$ Deep investigations should be done in order to understand the factors (physicochemical properties, environmental conditions, etc.) that influence these toxic effects. Moreover, nanogels could be inhaled, ingested, or skin-absorbed, coming into contact with genetic material. Thus, their toxicity should be studied not only during patient treatment but also during the entire manufacturing process and disposal. Manufacturing represents another big challenge. Indeed, the large-scale production of functionalized nanogels is necessary for their reproducible and consistent production. It is well-known that nanonetwork formation is much easier at the laboratory scale than at large scale, where bulk properties disfavor the formation of new surfaces. Consequently, scaling up laboratory or pilot technologies should be deeply investigated. These challenges include the possibility of controlling and predicting the proper formation of low concentrations of nanomaterials, aggregation, and the entire chemical process.

In addition, economic and financial barriers stand in the way of implementing nano-objects in general. Their high expense, in particular related to the functionalization of the starting materials, represents a high impediment for their clinical application. In order to justify these costs, they should prove to be a real breakthrough in the field.

\section{CONCLUSIONS}

Polymeric networks at the nanoscale represent a milestone for the efficient delivery of therapeutic molecules within different types of cells and interactions with living tissues. In particular, nanogels show significant advantages related to their carbonbased chemical composition, swelling/deswelling behavior, and ability to limit as much as possible the immune responses. However, the simple combination of synthetic and natural polymers does not always satisfy the biocompatible and curative criteria of the clinical applications. For these reasons, the functionalization of nanogels with specific and selective chemical groups guarantees the improvement of the final design: drugs, gene materials, peptides, and proteins can be loaded and released in the targeted region, in a desired intracellular area, preventing unwanted loss of the therapeutic cargo.

Nanogels can be used to limit under- and overdosing, promote controlled biomolecule release, increase patient compliance, and perform tunable cell uptake, improving their half-lives in biological compartments. As discussed in this review, the functionalization techniques allow not only the introduction of new chemical groups, different from the standard polymeric structures, but also conjugation with ligands, peptides, cell receptors, growth factors, and fluorescent agents that play a key role for the efficiency of the synthesized nanocarriers in all fields of application: the functionalization approaches appear as the basic line of thinking for the progress in the treatment of disorders and satisfaction of therapeutic needs.

\section{AUTHOR INFORMATION}

\section{Corresponding Author}

*Tel.: +39 0223993145. Fax: +39 0223993180. E-mail: filippo. rossi@polimi.it.

ORCID

Emanuele Mauri: 0000-0002-6203-8405

Filippo Rossi: 0000-0003-2665-120X

Notes

The authors declare no competing financial interest.

\section{ACKNOWLEDGMENTS}

G.P.'s contribution was partially supported by the European Prosafe JTC-2016 R\&D Project Grant "GoNanoBioMat" and Swiss KTI Grant 19267.1-PFNM.NM.

\section{REFERENCES}

(1) Buwalda, S. J.; Vermonden, T.; Hennink, W. E. Hydrogels for Therapeutic Delivery: Current Developments and Future Directions. Biomacromolecules 2017, 18, 316-330.

(2) Prasad, M.; Lambe, U. P.; Brar, B.; Shah, I.; J, M.; Ranjan, K.; Rao, R.; Kumar, S.; Mahant, S.; Khurana, S. K.; Iqbal, H. M. N.; Dhama, K.; Misri, J.; Prasad, G. Nanotherapeutics: An Insight into Healthcare and Multi-dimensional Applications in Medical Sector of the Modern World. Biomed. Pharmacother. 2018, 97, 1521-1537.

(3) Re, F.; Gregori, M.; Masserini, M. Nanotechnology for Neurodegenerative Disorders. Nanomedicine 2012, 8, S51-S58.

(4) Sivaram, A. J.; Rajitha, P.; Maya, S.; Jayakumar, R.; Sabitha, M. Nanogels for Delivery, Imaging and Therapy. Wires Nanomed. Nanobiotechnol. 2015, 7, 509-533.

(5) Liu, G. Y.; An, Z. S. Frontiers in the Design and Synthesis of Advanced Nanogels for Nanomedicine. Polym. Chem. 2014, 5, 15591565.

(6) Baipaywad, P.; Udomluck, N.; Pyo, S. G.; Park, H. H.; Park, H. Fabrication of Nanogels for Delivery of Molecules. J. Nanosci. Nanotechnol. 2014, 14, 7363-7373.

(7) Ghorbani, M.; Hamishehkar, H. Redox-responsive Smart Nanogels for Intracellular Targeting of Therapeutic Agents: Applications and Recent Advances. J. Drug Target 2018, 6, 1-15.

(8) Neamtu, I.; Rusu, A. G.; Diaconu, A.; Nita, L. E.; Chiriac, A. P. Basic Concepts and Recent Advances in Nanogels as Carriers for Medical Applications. Drug Delivery 2017, 24, 539-557.

(9) Asadian-Birjand, M.; Sousa-Herves, A.; Steinhilber, D.; Cuggino, J. C.; Calderon, M. Functional Nanogels for Biomedical Applications. Curr. Med. Chem. 2012, 19, 5029-5043.

(10) de Sousa, M.; Visani de Luna, L. A.; Fonseca, L. C.; Giorgio, S.; Alves, O. L. Folic-Acid-Functionalized Graphene Oxide Nanocarrier: 
Synthetic Approaches, Characterization, Drug Delivery Study, and Antitumor Screening. ACS Appl. Nano Mater. 2018, 1, 922-932.

(11) Rolland, J. P.; Maynor, B. W.; Euliss, L. E.; Exner, A. E.; Denison, G. M.; DeSimone, J. M. Direct Fabrication and Harvesting of Monodisperse, Shape-specific Nanobiomaterials. J. Am. Chem. Soc. 2005, 127, 10096-10100.

(12) Kersey, F. R.; Merkel, T. J.; Perry, J. L.; Napier, M. E.; DeSimone, J. M. Effect of Aspect Ratio and Deformability on Nanoparticle Extravasation through Nanopores. Langmuir 2012, 28, 8773-8781.

(13) Vasudevan, M.; Buse, E.; Lu, D. L.; Krishna, H.; Kalyanaraman, R.; Shen, A. Q.; Khomami, B.; Sureshkumar, R. Irreversible nanogel formation in surfactant solutions by microporous flow. Nat. Mater. 2010, 9, 436-441.

(14) Vinogradov, S.; Batrakova, E.; Kabanov, A. Poly(ethylene glycol)-polyethyleneimine NanoGel (TM) Particles: Novel Drug Delivery Systems for Antisense Oligonucleotides. Colloids Surf., B 1999, 16, 291-304.

(15) Lemieux, P.; Vinogradov, S. V.; Gebhart, C. L.; Guerin, N.; Paradis, G.; Nguyen, H. K.; Ochietti, B.; Suzdaltseva, Y. G.; Bartakova, E. V.; Bronich, T. K.; St-Pierre, Y.; Alakhov, V. Y.; Kabanov, A. V. Block and Graft Copolymers and Nanogel (TM) Copolymer Networks for DNA Delivery into Cell. J. Drug Target 2000, 8, 91-105.

(16) Akiyoshi, K.; Deguchi, S.; Moriguchi, N.; Yamaguchi, S.; Sunamoto, J. Self-Aggregates of Hydrophobized Polysaccharides in Water-Formation and Characteristics of Nanoparticles. Macromolecules 1993, 26, 3062-3068.

(17) Amamoto, Y.; Kikuchi, M.; Masunaga, H.; Ogawa, H.; Sasaki, S.; Otsuka, H.; Takahara, A. Mesh-size Control and Functionalization of Reorganizable Chemical Gels by Monomer Insertion into their Crosslinking Points. Polym. Chem. 2011, 2, 957-962.

(18) Soni, K. S.; Desale, S. S.; Bronich, T. K. Nanogels: An Overview of Properties, Biomedical Applications and Obstacles to Clinical Translation. J. Controlled Release 2016, 240, 109-126.

(19) Ryu, J. H.; Chacko, R. T.; Jiwpanich, S.; Bickerton, S.; Babu, R. P.; Thayumanavan, S. Self-Cross-Linked Polymer Nanogels: A Versatile Nanoscopic Drug Delivery Platform. J. Am. Chem. Soc. 2010, 132, 17227-17235.

(20) Sasaki, Y.; Akiyoshi, K. Nanogel Engineering for New Nanobiomaterials: From Chaperoning Engineering to Biomedical Applications. Chem. Rec. 2010, 10, 366-376.

(21) Sood, N.; Bhardwaj, A.; Mehta, S.; Mehta, A. Stimuli-responsive Hydrogels in Drug Delivery and Tissue Engineering. Drug Delivery 2016, 23, 748-770

(22) Lalitha, K.; Prasad, Y. S.; Maheswari, C. U.; Sridharan, V.; John, G.; Nagarajan, S. Stimuli Responsive Hydrogels Derived from a Renewable Resource: Synthesis, Self-assembly in water and Application in Drug Delivery. J. Mater. Chem. B 2015, 3, 5560-5568.

(23) Raemdonck, K.; Demeester, J.; De Smedt, S. Advanced Nanogel Engineering for Drug Delivery. Soft Matter 2009, 5, 707-715.

(24) Eckmann, D. M.; Composto, R. J.; Tsourkas, A.; Muzykantov, V. R. Nanogel Carrier Design for Targeted Drug Delivery. J. Mater. Chem. B 2014, 2, 8085-8097.

(25) Blackburn, W. H.; Lyon, L. A. Size-controlled Synthesis of Monodisperse Core/shell Nanogels. Colloid Polym. Sci. 2008, 286, $563-569$.

(26) Akiyama, E.; Morimoto, N.; Kujawa, P.; Ozawa, Y.; Winnik, F. M.; Akiyoshi, K. Self-assembled nanogels of cholesteryl-modified polysaccharides: Effect of the Polysaccharide Structure on their Association Characteristics in the Dilute and Semidilute Regimes. Biomacromolecules 2007, 8, 2366-2373.

(27) Bencherif, S. A.; Siegwart, D. J.; Srinivasan, A.; Horkay, F.; Hollinger, J. O.; Washburn, N. R.; Matyjaszewski, K. Nanostructured Hybrid Hydrogels Prepared by a Combination of Atom Transfer Radical Polymerization and Free Radical Polymerization. Biomaterials 2009, 30, 5270-5278.

(28) Smith, M. H.; Lyon, L. A. Multifunctional Nanogels for siRNA Delivery. Acc. Chem. Res. 2012, 45, 985-993.
(29) Merino, S.; Martin, C.; Kostarelos, K.; Prato, M.; Vazquez, E. Nanocomposite Hydrogels: 3D Polymer-Nanoparticle Synergies for On-Demand Drug Delivery. ACS Nano 2015, 9, 4686-4697.

(30) Wu, W. T.; Mitra, N.; Yan, E. C. Y.; Zhou, S. Q. Multifunctional Hybrid Nanogel for Integration of Optical Glucose Sensing and SelfRegulated Insulin Release at Physiological pH. ACS Nano 2010, 4, 4831-4839.

(31) Aguirre, G.; Villar-Alvarez, E.; Gonzalez, A.; Ramos, J.; Taboada, P.; Forcada, J. Biocompatible Stimuli-Responsive Nanogels for Controlled Antitumor Drug Delivery. J. Polym. Sci., Part A: Polym. Chem. 2016, 54, 1694-1705.

(32) Zha, L. S.; Banik, B.; Alexis, F. Stimulus Responsive Nanogels for Drug Delivery. Soft Matter 2011, 7, 5908-5916.

(33) Li, Y.; Bui, Q. N.; Duy, L. T. M.; Yang, H. Y.; Lee, D. S. One-Step Preparation of $\mathrm{pH}$-Responsive Polymeric Nanogels as Intelligent Drug Delivery Systems for Tumor Therapy. Biomacromolecules 2018, 19, 2062-2070.

(34) Wei, X.; Senanayake, T. H.; Warren, G.; Vinogradov, S. V. Hyaluronic Acid-Based Nanogel-Drug Conjugates with Enhanced Anticancer Activity Designed for the Targeting of CD44-Positive and Drug-Resistant Tumors. Bioconjugate Chem. 2013, 24, 658-668.

(35) Chen, W.; Achazi, K.; Schade, B.; Haag, R. Charge-Conversional and Reduction-Sensitive Poly(vinyl alcohol) Nanogels for Enhanced Cell Uptake and Efficient Intracellular Doxorubicin Release. J. Controlled Release 2015, 205, 15-24.

(36) Manju, S.; Sreenivasan, K. Enhanced Drug Loading on Magnetic Nanoparticles by Layer-by-Layer Assembly Using Drug Conjugates: Blood Compatibility Evaluation and Targeted Drug Delivery in Cancer Cells. Langmuir 2011, 27, 14489-14496.

(37) Holback, H.; Yeo, Y.; Park, K. Hydrogel swelling behavior and its biomedical applications. In Biomedical Hydrogels; Rimmer, S., Ed.; Woodhead Publishing: Cambridge, U.K., 2011; Chapter 1, pp 3-24.

(38) Lin, H.; Dan, W. H.; Dan, N. H. The Water State in Crosslinked Poly(vinyl alcohol)-Collagen Hydrogel and its Swelling Behavior. J. Appl. Polym. Sci. 2012, 123, 2753-2761.

(39) Brannonpeppas, L.; Peppas, N. A. Equilibrium Swelling Behavior of Ph-Sensitive Hydrogels. Chem. Eng. Sci. 1991, 46, 715-722.

(40) Yang, H. E.; Bae, Y. C. Group Contribution Method for the Swelling Behavior of Thermo-Responsive Hydrogels. J. Polym. Sci., Part B: Polym. Phys. 2017, 55, 455-463.

(41) Pikabea, A.; Ramos, J.; Forcada, J. Production of Cationic Nanogels with Potential Use in Controlled Drug Delivery. Part. Part. Syst. Char. 2014, 31, 101-109.

(42) Cuenot, S.; Radji, S.; Alem, H.; Demoustier-Champagne, S.; Jonas, A. M. Control of Swelling of Responsive Nanogels by Nanoconfinement. Small 2012, 8, 2978-2985.

(43) Kim, E.; Lee, J.; Kim, D.; Lee, K. E.; Han, S. S.; Lim, N.; Kang, J.; Park, C. G.; Kim, K. Solvent-Responsive Polymer Nanocapsules with Controlled Permeability: Encapsulation and Release of a Fluorescent Dye by Swelling and Deswelling. Chem. Commun. 2009, 1, 1472-1474.

(44) Nukolova, N. V.; Yang, Z. G.; Kim, J. O.; Kabanov, A. V.; Bronich, T. K. Polyelectrolyte Nanogels Decorated with Monoclonal Antibody for Targeted Drug Delivery. React. Funct. Polym. 2011, 71, 315-323.

(45) Qiu, L. P.; Qiao, M. X.; Chen, Q.; Tian, C. M.; Long, M. M.; Wang, M. Y.; Li, Z.; Hu, W.; Li, G.; Cheng, L.; Cheng, L. F.; Hu, H. Y.; Zhao, X. L.; Chen, D. W. Enhanced Effect of pH-Sensitive Mixed Copolymer Micelles for Overcoming Multidrug Resistance of Doxorubicin. Biomaterials 2014, 35, 9877-9887.

(46) Kono, K.; Takashima, M.; Yuba, E.; Harada, A.; Hiramatsu, Y.; Kitagawa, H.; Otani, T.; Maruyama, K.; Aoshima, S. Multifunctional Liposomes having Target Specificity, Temperature-Triggered Release, and Near-Infrared Fluorescence Imaging for Tumor-Specific Chemotherapy. J. Controlled Release 2015, 216, 69-77.

(47) Napier, M. E.; Desimone, J. M. Nanoparticle Drug Delivery Platform. Polym. Rev. 2007, 47, 321-327.

(48) Wu, H. Q.; Wang, C. C. Biodegradable Smart Nanogels: A New Platform for Targeting Drug Delivery and Biomedical Diagnostics. Langmuir 2016, 32, 6211-6225. 
(49) An, Z. S.; Qiu, Q.; Liu, G. Y. Synthesis of Architecturally welldefined Nanogels via RAFT Polymerization for Potential Bioapplications. Chem. Commun. 2011, 47, 12424-12440.

(50) Nayak, S.; Lyon, L. A. Soft Nanotechnology with Soft Nanoparticles. Angew. Chem., Int. Ed. 2005, 44, 7686-7708.

(51) Zhang, L.; Cao, Z. Q.; Li, Y. T.; Ella-Menye, J. R.; Bai, T.; Jiang, S. Y. Softer Zwitterionic Nanogels for Longer Circulation and Lower Splenic Accumulation. ACS Nano 2012, 6, 6681-6686.

(52) Hendrickson, G. R.; Lyon, L. A. Microgel Translocation through Pores under Confinement. Angew. Chem., Int. Ed. 2010, 49, 21932197.

(53) Holden, D. A.; Hendrickson, G.; Lyon, L. A.; White, H. S. Resistive Pulse Analysis of Microgel Deformation During Nanopore Translocation. J. Phys. Chem. C 2011, 115, 2999-3004.

(54) Hamidi, M.; Azadi, A.; Rafiei, P. Hydrogel Nanoparticles in Drug Delivery. Adv. Drug Delivery Rev. 2008, 60, 1638-1649.

(55) Chacko, R. T.; Ventura, J.; Zhuang, J. M.; Thayumanavan, S. Polymer nanogels: A versatile Nanoscopic Drug Delivery Platform. Adv. Drug Delivery Rev. 2012, 64, 836-851.

(56) Sanson, N.; Rieger, J. Synthesis of Nanogels/Microgels by Conventional and Controlled Radical Crosslinking Copolymerization. Polym. Chem. 2010, 1, 965-977.

(57) Li, X. T.; Li, X. X.; Shi, X. D.; Qiu, G.; Lu, X. H. Thermosensitive DEA/DMA Copolymer Nanogel: Low Initiator Induced Synthesis and Structural Colored Colloidal Array's Optical Properties. Eur. Polym. J. 2017, 96, 484-493.

(58) Sumerlin, B. S. Proteins as Initiators of Controlled Radical Polymerization: Grafting-from via ATRP and RAFT. ACS Macro Lett. 2012, 1, 141-145.

(59) Amamoto, Y.; Kikuchi, M.; Otsuka, H.; Takahara, A. ArmReplaceable Star-like Nanogels: Arm Detachment and Arm Exchange Reactions by Dynamic Covalent Exchanges of Alkoxyamine Units. Polym. J. 2010, 42, 860-867.

(60) Zhang, Y.; Ding, J. X.; Li, M. Q.; Chen, X.; Xiao, C. S.; Zhuang, X. L.; Huang, Y. B.; Chen, X. S. One-Step "Click Chemistry"-Synthesized Cross-Linked Prodrug Nanogel for Highly Selective Intracellular Drug Delivery and Upregulated Antitumor Efficacy. ACS Appl. Mater. Interfaces 2016, 8, 10673-10682.

(61) Liu, J. C.; Uhlir, C.; Shah, P. K.; Sun, F.; Stansbury, J. W. Controlled Nanogel and Macrogel Structures from Self-Assembly of a Stimuli-Responsive Amphiphilic Block Copolymer. RSC Adv. 2016, 6, 64791-64798.

(62) Xing, T.; Lai, B.; Ye, X. D.; Yan, L. F. Disulfide Core CrossLinked PEGylated Polypeptide Nanogel Prepared by a One-Step Ring Opening Copolymerization of N-Carboxyanhydrides for Drug Delivery. Macromol. Biosci. 2011, 11, 962-969.

(63) Sun, W. H.; An, Z. S.; Wu, P. Y. Revealing the Distinct Thermal Transition Behavior between PEGA-based Linear Polymers and their Disulfide Cross-Linked Nanogels. Phys. Chem. Chem. Phys. 2017, 19, 25746-25753.

(64) Gyarmati, B.; Nemethy, A.; Szilagyi, A. Reversible Disulphide Formation in Polymer Networks: A Versatile Functional Group from Synthesis to Applications. Eur. Polym. J. 2013, 49, 1268-1286.

(65) Sarika, P. R.; Anil Kumar, P. R.; Raj, D. K.; James, N. R. Nanogels Based on Alginic Aldehyde and Gelatin by Inverse Miniemulsion Technique: Synthesis and Characterization. Carbohydr. Polym. 2015, $119,118-125$.

(66) Su, H. Y.; Jia, Q. M.; Shan, S. S. Synthesis and Characterization of Schiff base Contained Dextran Microgels in Water-in-Oil Inverse Microemulsion. Carbohydr. Polym. 2016, 152, 156-162.

(67) Pioge, S.; Nesterenko, A.; Brotons, G.; Pascual, S.; Fontaine, L.; Gaillard, C.; Nicol, E. Core Cross-Linking of Dynamic Diblock Copolymer Micelles: Quantitative Study of Photopolymerization Efficiency and Micelle Structure. Macromolecules 2011, 44, 594-603.

(68) Choi, J. B.; Park, J. S.; Khil, M. S.; Gwon, H. J.; Lim, Y. M.; Jeong, S. I.; Shin, Y. M.; Nho, Y. C. Characterization and Antimicrobial Property of Poly(Acrylic Acid) Nanogel Containing Silver Particle Prepared by Electron Beam. Int. J. Mol. Sci. 2013, 14, 11011-11023.
(69) Du, J. Z.; Sun, T. M.; Song, W. J.; Wu, J.; Wang, J. A TumorAcidity-Activated Charge-Conversional Nanogel as an Intelligent Vehicle for Promoted Tumoral-Cell Uptake and Drug Delivery. Angew. Chem., Int. Ed. 2010, 49, 3621-3626.

(70) Song, L.; Liang, X.; Yang, S.; Wang, N.; He, T.; Wang, Y.; Zhang, L.; Wu, Q.; Gong, C. Novel Polyethyleneimine-R8-Heparin Nanogel for High-Efficiency Gene Delivery in Vitro and in Vivo. Drug Delivery 2018, 25, 122-131.

(71) Knipe, J. M.; Strong, L. E.; Peppas, N. A. Enzyme- and pHResponsive Microencapsulated Nanogels for Oral Delivery of siRNA to Induce TNF-alpha Knockdown in the Intestine. Biomacromolecules 2016, 17, 788-797.

(72) Zeng, Z. P.; She, Y. Q.; Peng, Z. P.; Wei, J. C.; He, X. H. EnzymeMediated in Situ Formation of $\mathrm{pH}$-sensitive Nanogels for Proteins Delivery. RSC Adv. 2016, 6, 8032-8042.

(73) Petr, S.; Jana, D.; Peter, C.; Ewa, P.; Vladimír, P. Poly(amino acid)-based nanogel by horseradish peroxidase catalyzed crosslinking in an inverse miniemulsion. Colloid Polym. Sci. 2018, 296, 995-1003.

(74) Zhang, X. J.; Malhotra, S.; Molina, M.; Haag, R. Micro- and Nanogels with Labile Crosslinks - from Synthesis to Biomedical Applications. Chem. Soc. Rev. 2015, 44, 1948-1973.

(75) Huppertz, T.; de Kruif, C. G. Structure and Stability of Nanogel Particles Prepared by Internal Cross-Linking of Casein Micelles. Int. Dairy J. 2008, 18, 556-565.

(76) Elsabahy, M.; Heo, G. S.; Lim, S. M.; Sun, G. R.; Wooley, K. L. Polymeric Nanostructures for Imaging and Therapy. Chem. Rev. 2015, $115,10967-11011$

(77) Tamura, M.; Ichinohe, S.; Tamura, A.; Ikeda, Y.; Nagasaki, Y. In Vitro and in Vivo Characteristics of Core-Shell Type Nanogel Particles: Optimization of Core Cross-Linking Density and Surface Poly(ethylene glycol) Density in PEGylated Nanogels. Acta Biomater. 2011, 7, 3354-3361.

(78) Chan, M.; Almutairi, A. Nanogels as Imaging Agents for Modalities Spanning the Electromagnetic Spectrum. Mater. Horiz. 2016, 3, 21-40.

(79) Oh, J. K.; Lee, D. I.; Park, J. M. Biopolymer-based Microgels/ Nanogels for Drug Delivery Applications. Prog. Polym. Sci. 2009, 34, $1261-1282$

(80) Sawada, S. I.; Yukawa, H.; Takeda, S.; Sasaki, Y.; Akiyoshi, K. Self-Assembled Nanogel of Cholesterol-Bearing Xyloglucan as a Drug Delivery Nanocarrier. J. Biomater. Sci., Polym. Ed. 2017, 28, 1183-1198.

(81) Shimoda, A.; Sawada, S.; Kano, A.; Maruyama, A.; Moquin, A.; Winnik, F. M.; Akiyoshi, K. Dual Crosslinked Hydrogel Nanoparticles by Nanogel Bottom-up Method for Sustained-Release Delivery. Colloids Surf., B 2012, 99, 38-44.

(82) Morimoto, N.; Hirano, S.; Takahashi, H.; Loethen, S.; Thompson, D. H.; Akiyoshi, K. Self-Assembled pH-Sensitive Cholesteryl Pullulan Nanogel As a Protein Delivery Vehicle. Biomacromolecules 2013, 14, 56-63.

(83) Xu, J.; Wong, D. H. C.; Byrne, J. D.; Chen, K.; Bowerman, C.; DeSimone, J. M. Future of the Particle Replication in Nonwetting Templates (PRINT) Technology. Angew. Chem., Int. Ed. 2013, 52, 6580-6589.

(84) Galloway, A. L.; Murphy, A.; DeSimone, J. M.; Di, J.; Herrmann, J. P.; Hunter, M. E.; Kindig, J. P.; Malinoski, F. J.; Rumley, M. A.; Stoltz, D. M.; Templeman, T. S.; Hubby, B. Development of a NanoparticleBased Influenza Vaccine using the PRINT (R) Technology. Nanomedicine 2013, 9, 523-531.

(85) Sambade, M.; Deal, A.; Schorzman, A.; Luft, J. C.; Bowerman, C.; Chu, K.; Karginova, O.; Van Swearingen, A.; Zamboni, W.; DeSimone, J.; Anders, C. K. Efficacy and Pharmacokinetics of a Modified AcidLabile Docetaxel-PRINT (R) Nanoparticle Formulation against NonSmall-Cell Lung Cancer Brain Metastases. Nanomedicine 2016, 11, 1947-1955.

(86) Takeuchi, T.; Kitayama, Y.; Sasao, R.; Yamada, T.; Toh, K.; Matsumoto, Y.; Kataoka, K. Molecularly Imprinted Nanogels Acquire Stealth In Situ by Cloaking Themselves with Native Dysopsonic Proteins. Angew. Chem., Int. Ed. 2017, 56, 7088-7092. 
(87) Culver, H. R.; Sharma, I.; Wechsler, M. E.; Anslyn, E. V.; Peppas, N. A. Charged Poly $(\mathrm{N}$-isopropylacrylamide) Nanogels for use as Differential Protein Receptors in a Turbidimetric Sensor Array. Analyst 2017, 142, 3183-3193.

(88) Motornov, M.; Roiter, Y.; Tokarev, I.; Minko, S. StimuliResponsive Nanoparticles, Nanogels and Capsules for Integrated Multifunctional Intelligent Systems. Prog. Polym. Sci. 2010, 35, 174211.

(89) Qian, H. Q.; Wang, X.; Yuan, K. J.; Xie, C.; Wu, W.; Jiang, X. Q.; $\mathrm{Hu}, \mathrm{L}$. J. Delivery of Doxorubicin in Vitro and in Vivo using BioReductive Cellulose Nanogels. Biomater. Sci. 2014, 2, 220-232.

(90) Mauri, E.; Papa, S.; Masi, M.; Veglianese, P.; Rossi, F. Novel Functionalization Strategies to Improve Drug Delivery from Polymers. Expert Opin. Drug Delivery 2017, 14, 1305-1313.

(91) Blasco, E.; Sims, M. B.; Goldmann, A. S.; Sumerlin, B. S.; BarnerKowollik, C. 50th Anniversary Perspective: Polymer Functionalization. Macromolecules 2017, 50, 5215-5252.

(92) Sacchetti, A.; Mauri, E.; Sani, M.; Masi, M.; Rossi, F. MicrowaveAssisted Synthesis and Click Chemistry as Simple and Efficient Strategy for RGD Functionalized Hydrogels. Tetrahedron Lett. 2014, 55, 68176820.

(93) Jiang, Y. J.; Chen, J.; Deng, C.; Suuronen, E. J.; Zhong, Z. Y. Click Hydrogels, Microgels and Nanogels: Emerging Platforms for Drug Delivery and Tissue Engineering. Biomaterials 2014, 35, 4969-4985.

(94) Yi, G.; Son, J.; Yoo, J.; Park, C.; Koo, H. Application of Click Chemistry in Nanoparticle Modification and its Targeted Delivery. Biomater. Res. 2018, 22, 13.

(95) Tatiparti, K.; Sau, S.; Gawde, K. A.; Iyer, A. K. Copper-Free "Click" Chemistry-Based Synthesis and Characterization of Carbonic Anhydrase-IX Anchored Albumin-Paclitaxel Nanoparticles for Targeting Tumor Hypoxia. Int. J. Mol. Sci. 2018, 19, 838.

(96) Kade, M. J.; Burke, D. J.; Hawker, C. J. The Power of Thiol-ene Chemistry. J. Polym. Sci., Part A: Polym. Chem. 2010, 48, 743-750.

(97) Lowe, A. B. Thiol-Ene "Click" Reactions and Recent Applications in Polymer and Materials Synthesis: a First Update. Polym. Chem. 2014, 5, 4820-4870.

(98) Hoyle, C. E.; Lowe, A. B.; Bowman, C. N. Thiol-Click Chemistry: a Multifaceted Toolbox for Small Molecule and Polymer Synthesis. Chem. Soc. Rev. 2010, 39, 1355-1387.

(99) Ferruti, P.; Bianchi, S.; Ranucci, E.; Chiellini, F.; Caruso, V. Novel Poly(amido-amine)-based Hydrogels as Scaffolds for Tissue Engineering. Macromol. Biosci. 2005, 5, 613-622.

(100) Vernon, B.; Tirelli, N.; Bachi, T.; Haldimann, D.; Hubbell, J. A. Water-borne, in Situ Crosslinked Biomaterials from Phase-Segregated Precursors. J. Biomed. Mater. Res., Part A 2003, 64A, 447-456.

(101) Biedermann, F.; Appel, E. A.; del Barrio, J.; Gruendling, T.; Barner-Kowollik, C.; Scherman, O. A. Postpolymerization Modification of Hydroxyl-Functionalized Polymers with Isocyanates. Macromolecules 2011, 44, 4828-4835.

(102) Sun, G. M.; Chu, C. C. Synthesis, Characterization of Biodegradable Dextran-Allyl Isocyanate-Ethylamine/Polyethylene Glycol-Diacrylate Hydrogels and their in Vitro Release of Albumin. Carbohydr. Polym. 2006, 65, 273-287.

(103) Kathalewar, M. S.; Joshi, P. B.; Sabnis, A. S.; Malshe, V. C. NonIsocyanate Polyurethanes: from Chemistry to Applications. RSC Adv. 2013, 3, 4110-4129.

(104) Jackson, A. W.; Stakes, C.; Fulton, D. A. The Formation of Core Cross-linked Star Polymer and Nanogel Assemblies Facilitated by the Formation of Dynamic Covalent Imine Bonds. Polym. Chem. 2011, 2, 2500-2511.

(105) Mukherjee, S.; Bapat, A. P.; Hill, M. R.; Sumerlin, B. S. Oximes as Reversible Links in Polymer Chemistry: Dynamic Macromolecular Stars. Polym. Chem. 2014, 5, 6923-6931.

(106) Foster, J. C.; Matson, J. B. Functionalization of Methacrylate Polymers with Thiooximes: A Robust Postpolymerization Modification Reaction and a Method for the Preparation of H2S-Releasing Polymers. Macromolecules 2014, 47, 5089-5095.
(107) Pikabea, A.; Forcada, J. Novel Approaches for the Preparation of Magnetic Nanogels via Covalent Bonding. J. Polym. Sci., Part A: Polym. Chem. 2017, 55, 3573-3586.

(108) Pehlivaner Kara, M. O.; Ekenseair, A. K. Free Epoxide Content Mediates Encapsulated Cell Viability and Activity through Protein Interactions in a Thermoresponsive, In Situ Forming Hydrogel. Biomacromolecules 2017, 18, 1473-1481.

(109) Akhtar, R.; Naqvi, S. A. R.; Zahoor, A. F.; Saleem, S. Nucleophilic Ring Opening Reactions of Aziridines. Mol. Diversity 2018, 22, 447-501.

(110) Roehn, U.; Becaud, J.; Mu, L. J.; Srinivasan, A.; Stellfeld, T.; Fitzner, A.; Graham, K.; Dinkelborg, L.; Schubiger, A. P.; Ametamey, S. M. Nucleophilic Ring-Opening of Activated Aziridines: A One-Step Method for Labeling Biomolecules with Fluorine-18. J. Fluorine Chem. 2009, 130, 902-912.

(111) Carter, M. C. D.; Jennings, J.; Appadoo, V.; Lynn, D. M. Synthesis and Characterization of Backbone Degradable AzlactoneFunctionalized Polymers. Macromolecules 2016, 49, 5514-5526.

(112) Buck, M. E.; Lynn, D. M. Azlactone-Functionalized Polymers as Reactive Platforms for the Design of Advanced Materials: Progress in the Last Ten Years. Polym. Chem. 2012, 3, 66-80.

(113) Pan, Y.; Ma, L. J.; Lin, S.; Zhang, Y. F.; Cheng, B. W.; Meng, J. Q. One-Step Bimodel Grafting via a Multicomponent Reaction toward Antifouling and Antibacterial TFC RO Membranes. J. Mater. Chem. A 2016, 4, 15945-15960.

(114) Mironov, M. A.; Shulepov, I. D.; Ponomarev, V. S.; Bakulev, V. A. Synthesis of Polyampholyte Microgels from Colloidal Salts of Pectinic Acid and their Application as pH-Responsive Emulsifiers. Colloid Polym. Sci. 2013, 291, 1683-1691.

(115) Tan, J. P. K.; Tan, M. B. H.; Tam, M. K. C. Application of Nanogel Systems in the Administration of Local Anesthetics. Local Reg. Anesth. 2010, 3, 93-100.

(116) Chambre, L.; Degirmenci, A.; Sanyal, R.; Sanyal, A. MultiFunctional Nanogels as Theranostic Platforms: Exploiting Reversible and Nonreversible Linkages for Targeting, Imaging, and Drug Delivery. Bioconjugate Chem. 2018, 29, 1885-1896.

(117) Kgesa, T.; Choonara, Y. E.; Tyagi, C.; Tomar, L. K.; Kumar, P.; du Toit, L. C.; Pillay, V. Disulphide-thiol chemistry: A Multi-faceted Tool for Macromolecular Design and Synthesis of Polyfunctional Materials for Specialized Drug Delivery. Curr. Drug Delivery 2015, 12, 282-298.

(118) Shimoda, A.; Sawada, S.-i.; Akiyoshi, K. Cell Specific PeptideConjugated Polysaccharide Nanogels for Protein Delivery. Macromol. Biosci. 2011, 11, 882-888.

(119) Fisher, O. Z.; Kim, T.; Dietz, S. R.; Peppas, N. A. Enhanced Core Hydrophobicity, Functionalization and Cell Penetration of Polybasic Nanomatrices. Pharm. Res. 2009, 26, 51-60.

(120) Chen, J. J.; Ding, J. X.; Xu, W. G.; Sun, T. M.; Xiao, H. H.; Zhuang, X. L.; Chen, X. S. Receptor and Microenvironment DualRecognizable Nanogel for Targeted Chemotherapy of Highly Metastatic Malignancy. Nano Lett. 2017, 17, 5180-5180.

(121) Fang, J.; Nakamura, H.; Maeda, H. The EPR effect: Unique Features of Tumor Blood Vessels for Drug Delivery, Factors involved, and Limitations and Augmentation of the Effect. Adv. Drug Delivery Rev. 2011, 63, 136-151.

(122) Tibbitt, M. W.; Dahlman, J. E.; Langer, R. Emerging Frontiers in Drug Delivery. J. Am. Chem. Soc. 2016, 138, 704-717.

(123) Park, K. Controlled Drug Delivery Aystems: Past forward and Future Back. J. Controlled Release 2014, 190, 3-8.

(124) Rossi, F.; Perale, G.; Papa, S.; Forloni, G.; Veglianese, P. Current Options for Drug Delivery to the Spinal Cord. Expert Opin. Drug Delivery 2013, 10, 385-396.

(125) Peppas, N. A. Historical Perspective on Advanced Drug Delivery: How Engineering Design and Mathematical Modeling helped the Field Mature. Adv. Drug Delivery Rev. 2013, 65, 5-9.

(126) Azagarsamy, M. A.; Alge, D. L.; Radhakrishnan, S. J.; Tibbitt, M. W.; Anseth, K. S. Photocontrolled Nanoparticles for On-Demand Release of Proteins. Biomacromolecules 2012, 13, 2219-2224. 
(127) Nicolas, J.; Mura, S.; Brambilla, D.; Mackiewicz, N.; Couvreur, P. Design, Functionalization Strategies and Biomedical Applications of Targeted Biodegradable/Biocompatible Polymer-Based Nanocarriers for Drug Delivery. Chem. Soc. Rev. 2013, 42, 1147-1235.

(128) Posadas, I.; Monteagudo, S.; Cena, V. Nanoparticles for BrainSpecific Drug and Genetic Material Delivery, Imaging and Diagnosis. Nanomedicine 2016, 11, 833-849.

(129) Hou, L.; Zheng, Y. Z.; Wang, Y. C.; Hu, Y. R.; Shi, J. J.; Liu, Q.; Zhang, H. J.; Zhang, Z. Z. Self-Regulated Carboxyphenylboronic AcidModified Mesoporous Silica Nanoparticles with "Touch Switch" Releasing Property for Insulin Delivery. ACS Appl. Mater. Interfaces 2018, 10, 21927-21938.

(130) Pakulska, M. M.; Elliott Donaghue, I.; Obermeyer, J. M.; Tuladhar, A.; McLaughlin, C. K.; Shendruk, T. N.; Shoichet, M. S. Encapsulation-Free Controlled Release: Electrostatic Adsorption eliminates the Need for Protein Encapsulation in PLGA Nanoparticles. Sci. Adv. 2016, 2, No. e1600519.

(131) Chang, M. L.; Zhang, F.; Wei, T.; Zuo, T. T.; Guan, Y. Y.; Lin, G. M.; Shao, W. Smart Linkers in Polymer-Drug Conjugates for Tumor-Targeted Delivery. J. Drug Target 2016, 24, 475-491.

(132) Vulic, K.; Shoichet, M. S. Tunable Growth Factor Delivery from Injectable Hydrogels for Tissue Engineering. J. Am. Chem. Soc. 2012, $134,882-885$.

(133) Rossi, F.; van Griensven, M. Polymer Functionalization as a Powerful Tool to Improve Scaffold Performances. Tissue Eng., Part A 2014, 20, 2043-2051.

(134) Wu, W.; Yao, W.; Wang, X.; Xie, C.; Zhang, J. L.; Jiang, X. Q. Bioreducible Heparin-Based Nanogel Drug Delivery System. Biomaterials 2015, 39, 260-268.

(135) Ossipov, D.; Kootala, S.; Yi, Z. Y.; Yang, X.; Hilborn, J. Orthogonal Chemoselective Assembly of Hyaluronic Acid Networks and Nanogels for Drug Delivery. Macromolecules 2013, 46, 4105-4113.

(136) Beke, S.; Barenghi, R.; Farkas, B.; Romano, I.; Korosi, L.; Scaglione, S.; Brandi, F. Improved Cell Activity on Biodegradable Photopolymer Scaffolds using Titanate Nanotube Coatings. Mater. Sci. Eng., C 2014, 44, 38-43.

(137) Le, C. M. Q.; Cao, X. T.; Tu, T. T. K.; Gal, Y. S.; Lim, K. T. Facile Approach to Prepare $\mathrm{pH}$ and Redox-Responsive Nanogels via Diels-Alder Click Reaction. eXPRESS Polym. Lett. 2018, 12, 688-698.

(138) Li, C. T.; Huang, W.; Zhou, L. Z.; Huang, P.; Pang, Y.; Zhu, X. Y.; Yan, D. Y. PEGylated Poly(diselenide-phosphate) Nanogel as Efficient Self-Delivery Nanomedicine for Cancer Therapy. Polym. Chem. 2015, 6, 6498-6508.

(139) Adamo, G.; Grimaldi, N.; Sabatino, M. A.; Walo, M.; Dispenza, C.; Ghersi, G. E-beam Crosslinked Nanogels Conjugated with Monoclonal Antibodies in Targeting Strategies. Biol. Chem. 2017, 398, 277-287.

(140) Noree, S.; Tangpasuthadol, V.; Kiatkamjornwong, S.; Hoven, V. P. Cascade Post-Polymerization Modification of Single Pentafluorophenyl Ester-Bearing Homopolymer as a Facile Route to RedoxResponsive Nanogels. J. Colloid Interface Sci. 2017, 501, 94-102.

(141) Su, S. S.; Wang, H.; Liu, X. G.; Wu, Y.; Nie, G. J. iRGD-Coupled Responsive Fluorescent Nanogel for Targeted Drug Delivery. Biomaterials 2013, 34, 3523-3533.

(142) Yu, J. C.; Zhang, Y. Q.; Sun, W. J.; Wang, C.; Ranson, D.; Ye, Y. Q.; Weng, Y. Y.; Gu, Z. Internalized Compartments Encapsulated Nanogels for Targeted Drug Delivery. Nanoscale 2016, 8, 9178-9184.

(143) Mauri, E.; Veglianese, P.; Papa, S.; Mariani, A.; De Paola, M.; Rigamonti, R.; Chincarini, G. M. F.; Rimondo, S.; Sacchetti, A.; Rossi, F. Chemoselective Functionalization of Nanogels for Microglia Treatment. Eur. Polym. J. 2017, 94, 143-151.

(144) Pohlit, H.; Worm, M.; Langhanki, J.; Berger-Nicoletti, E.; Opatz, T.; Frey, H. Silver Oxide Mediated Monotosylation of Poly(ethylene glycol) (PEG): Heterobifunctional PEG via Polymer Desymmetrization. Macromolecules 2017, 50, 9196-9206.

(145) Pikabea, A.; Villar-Alvarez, E.; Forcada, J.; Taboada, P. pHControlled Doxorubicin Delivery from PDEAEMA-Based Nanogels. J. Mol. Liq. 2018, 266, 321-329.
(146) Jokerst, J. V.; Lobovkina, T.; Zare, R. N.; Gambhir, S. S. Nanoparticle PEGylation for Imaging and Therapy. Nanomedicine 2011, 6, 715-728.

(147) Sun, L.; Li, Q.; Zhang, L.; Xu, Z.; Kang, Y.; Xue, P. PEGylated Polydopamine Nanoparticles Incorporated with Indocyanine Green and Doxorubicin for Magnetically Guided Multimodal Cancer Therapy Triggered by Near-Infrared Light. ACS Appl. Nano Mater. 2018, 1, 325-336.

(148) Mauri, E.; Cappella, F.; Masi, M.; Rossi, F. PEGylation Influences Drug Delivery from Nanogels. J. Drug Delivery Sci. Technol. 2018, 46, 87-92.

(149) Spencer, D. S.; Luu, B. C.; Beckman, D. W.; Peppas, N. A. Control of Cationic Nanogel PEGylation in Heterogeneous ARGET ATRP Emulsion Polymerization with PEG Macromonomers. J. Polym. Sci., Part A: Polym. Chem. 2018, 56, 1536-1544.

(150) Park, D.; Kim, Y.; Kim, H.; Kim, K.; Lee, Y.-S.; Choe, J.; Hahn, J.-H.; Lee, H.; Jeon, J.; Choi, C.; Kim, Y.-M.; Jeoung, D. Hyaluronic Acid Promotes Angiogenesis by Inducing RHAMM-TGF $\beta$ Receptor Interaction via CD44-PKC $\delta$. Mol. Cells 2012, 33, 563-574.

(151) Yang, C. C.; Li, C.; Zhang, P.; Wu, W.; Jiang, X. Q. Redox Responsive Hyaluronic Acid Nanogels for Treating RHAMM (CD168) Over-expressive Cancer, both Primary and Metastatic Tumors. Theranostics 2017, 7, 1719-1734.

(152) Liang, K.; Ng, S.; Lee, F.; Lim, J.; Chung, J. E.; Lee, S. S.; Kurisawa, M. Targeted Intracellular Protein Delivery Based on Hyaluronic Acid-Green Tea Catechin Nanogels. Acta Biomater. 2016, 33, 142-152.

(153) Luo, D.; Saltzman, W. M. Synthetic DNA Delivery Systems. Nat. Biotechnol. 2000, 18, 33-37.

(154) Parra-Guillén, Z. P.; González-Aseguinolaza, G.; Berraondo, P.; Trocóniz, I. F. Gene Therapy: A Pharmacokinetic/Pharmacodynamic Modelling Overview. Pharm. Res. 2010, 27, 1487-1497.

(155) Karimi, M.; Ghasemi, A.; Sahandi Zangabad, P.; Rahighi, R.; Moosavi Basri, S. M.; Mirshekari, H.; Amiri, M.; Shafaei Pishabad, Z.; Aslani, A.; Bozorgomid, M.; Ghosh, D.; Beyzavi, A.; Vaseghi, A.; Aref, A. R.; Haghani, L.; Bahrami, S.; Hamblin, M. R. Smart Micro/ Nanoparticles in Stimulus-Responsive Drug/Gene Delivery Systems. Chem. Soc. Rev. 2016, 45, 1457-1501.

(156) Costa, D.; Valente, A. J. M.; Queiroz, J. Stimuli-Responsive Polyamine-DNA Blend Nanogels for Co-Delivery in Cancer Therapy. Colloids Surf., B 2015, 132, 194-201.

(157) Dimde, M.; Neumann, F.; Reisbeck, F.; Ehrmann, S.; CuellarCamacho, J. L.; Steinhilber, D.; Ma, N.; Haag, R. Defined pH-Sensitive Nanogels as Gene Delivery Platform for siRNA Mediated in Vitro Gene Silencing. Biomater. Sci. 2017, 5, 2328-2336.

(158) Lin, C.; Engbersen, J. F. J. Effect of Chemical Functionalities in Poly(amido amine)s for Non-Viral Gene Transfection. J. Controlled Release 2008, 132, 267-272.

(159) Jones, N. A.; Hill, I. R. C.; Stolnik, S.; Bignotti, F.; Davis, S. S.; Garnett, M. C. Polymer chemical structure is a key determinant of physicochemical and colloidal properties of polymer-DNA complexes for gene delivery. Biochim. Biophys. Acta, Gene Struct. Expression 2000, $1517,1-18$.

(160) Griffiths, P. C.; Paul, A.; Khayat, Z.; Wan, K. W.; King, S. M.; Grillo, I.; Schweins, R.; Ferruti, P.; Franchini, J.; Duncan, R. Understanding the Mechanism of Action of Poly(amidoamine)s as Endosomolytic Polymers: Correlation of Physicochemical and Biological Properties. Biomacromolecules 2004, 5, 1422-1427.

(161) Ferruti, P.; Manzoni, S.; Richardson, S. C. W.; Duncan, R.; Pattrick, N. G.; Mendichi, R.; Casolaro, M. Amphoteric Linear Poly(amido-amine)s as Endosomolytic Polymers: Correlation between Physicochemical and Biological Properties. Macromolecules 2000, 33, 7793-7800.

(162) Lin, C.; Zhong, Z.; Lok, M. C.; Jiang, X.; Hennink, W. E.; Feijen, J.; Engbersen, J. F. J. Linear Poly(amido amine)s with Secondary and Tertiary Amino Groups and Variable Amounts of Disulfide Linkages: Synthesis and in Vitro Gene Transfer Properties. J. Controlled Release 2006, 116, 130-137. 
(163) Zakeri, A.; Kouhbanani, M. A. J.; Beheshtkhoo, N.; Beigi, V.; Mousavi, S. M.; Hashemi, S. A. R.; Karimi Zade, A.; Amani, A. M.; Savardashtaki, A.; Mirzaei, E.; Jahandideh, S.; Movahedpour, A. Polyethylenimine-based Nanocarriers in Co-Delivery of Drug and Gene: a Developing Horizon. Nano Rev. Exp. 2018, 9, 1488497.

(164) Lai, W.-F.; Green, D. W.; Jung, H.-S. Linear Poly(ethylenimine) Cross-Linked by Methyl- $\beta$-Cyclodextrin for Gene Delivery. Curr. Gene Ther. 2014, 14, 258-268.

(165) Mimi, H.; Ho, K. M.; Siu, Y. S.; Wu, A.; Li, P. Polyethyleneimine-Based Core-Shell Nanogels: A Promising siRNA Carrier for Argininosuccinate Synthetase mRNA Knockdown in HeLa Cells. J. Controlled Release 2012, 158, 123-130.

(166) Park, J. S.; Yi, S. W.; Kim, H. J.; Park, K.-H. Receptor-Mediated Gene Delivery into Human Mesenchymal Stem Cells using Hyaluronic Acid-Shielded Polyethylenimine/pDNA Nanogels. Carbohydr. Polym. 2016, 136, 791-802.

(167) Hong, C. A.; Kim, J. S.; Lee, S. H.; Kong, W. H.; Park, T. G.; Mok, H.; Nam, Y. S. Reductively Dissociable siRNA-Polymer Hybrid Nanogels for Efficient Targeted Gene Silencing. Adv. Funct. Mater. 2013, 23, 316-322.

(168) Tamura, A.; Oishi, M.; Nagasaki, Y. Enhanced Cytoplasmic Delivery of siRNA Using a Stabilized Polyion Complex Based on PEGylated Nanogels with a Cross-Linked Polyamine Structure. Biomacromolecules 2009, 10, 1818-1827.

(169) Li, R.-Q.; Wu, W.; Song, H.-Q.; Ren, Y.; Yang, M.; Li, J.; Xu, F.-J. Well-Defined Reducible Cationic Nanogels Based on Functionalized Low-Molecular-Weight PGMA for Effective pDNA and siRNA Delivery. Acta Biomater. 2016, 41, 282-292.

(170) Moghimi, S. M.; Hunter, A. C.; Murray, J. C. Long-Circulating and Target-Specific Nanoparticles: Theory to Practice. Pharmacol. Rev. 2001, 53, 283-318

(171) Gajbhiye, K. R.; Gajbhiye, V.; Soni, V. Targeted Brain Delivery of Bioactive Molecules Using Nanocarriers. J. Bioequivalence Bioavailability 2015, 7, 112-122.

(172) Chen, Y.; Wilbon, P. A.; Zhou, J.; Nagarkatti, M.; Wang, C.; Chu, F.; Tang, C. Multifunctional Self-Fluorescent Polymer Nanogels for Label-free Imaging and Drug Delivery. Chem. Commun. 2013, 49, 297-299.

(173) Wang, D.; Wu, L.-P. Nanomaterials for Delivery of Nucleic Acid to the Central Nervous System (CNS). Mater. Sci. Eng., C 2017, 70, 1039-1046.

(174) Hwang, D. W.; Son, S.; Jang, J.; Youn, H.; Lee, S.; Lee, D.; Lee, Y.-S.; Jeong, J. M.; Kim, W. J.; Lee, D. S. A Brain-Targeted Rabies Virus Glycoprotein-Disulfide Linked PEI Nanocarrier for Delivery of Neurogenic MicroRNA. Biomaterials 2011, 32, 4968-4975.

(175) Vinogradov, S. V.; Batrakova, E. V.; Kabanov, A. V. Nanogels for Oligonucleotide Delivery to the Brain. Bioconjugate Chem. 2004, 15, $50-60$.

(176) Vinogradov, S. V.; Zeman, A. D.; Batrakova, E. V.; Kabanov, A. V. Polyplex Nanogel Formulations for Drug Delivery of Cytotoxic Nucleoside Analogs. J. Controlled Release 2005, 107, 143-157.

(177) Vinogradov, S. V.; Kohli, E.; Zeman, A. D. Cross-Linked Polymeric Nanogel Formulations of 5'-Triphosphates of Nucleoside Analogues: Role of the Cellular Membrane in Drug Release. Mol. Pharmaceutics 2005, 2, 449-461.

(178) Li, Y.; Maciel, D.; Rodrigues, J.; Shi, X.; Tomás, H. Biodegradable Polymer Nanogels for Drug/Nucleic Acid Delivery. Chem. Rev. 2015, 115, 8564-8608.

(179) Gao, Y.; Wang, Z.-Y.; Zhang, J.; Zhang, Y.; Huo, H.; Wang, T.; Jiang, T.; Wang, S. RVG-Peptide-Linked Trimethylated Chitosan for Delivery of siRNA to the Brain. Biomacromolecules 2014, 15, 10101018.

(180) Yang, H. N.; Choi, J. H.; Park, J. S.; Jeon, S. Y.; Park, K. D.; Park, K.-H. Differentiation of Endothelial Progenitor Cells into Endothelial Cells by Heparin-Modified Supramolecular Pluronic Nanogels Encapsulating bFGF and Complexed with VEGF165 Genes. Biomaterials 2014, 35, 4716-4728.

(181) Tam, P.; Monck, M.; Lee, D.; Ludkovski, O.; Leng, E. C.; Clow, K.; Stark, H.; Scherrer, P.; Graham, R. W.; Cullis, P. R. Stabilized
Plasmid-Lipid Particles for Systemic Gene Therapy. Gene Ther. 2000, 7, 1867-1874.

(182) Hatakeyama, H.; Akita, H.; Harashima, H. A multifunctional envelope type nano device (MEND) for gene delivery to tumours based on the EPR effect: A strategy for overcoming the PEG dilemma. Adv. Drug Delivery Rev. 2011, 63, 152-160.

(183) Nishimura, T.; Yamada, A.; Umezaki, K.; Sawada, S.-i.; Mukai, S.-a.; Sasaki, Y.; Akiyoshi, K. Self-Assembled Polypeptide Nanogels with Enzymatically Transformable Surface as a Small Interfering RNA Delivery Platform. Biomacromolecules 2017, 18, 3913-3923.

(184) Blackburn, W. H.; Dickerson, E. B.; Smith, M. H.; McDonald, J. F.; Lyon, L. A. Peptide-Functionalized Nanogels for Targeted siRNA Delivery. Bioconjugate Chem. 2009, 20, 960-968.

(185) Lux, J.; White, A. G.; Chan, M.; Anderson, C. J.; Almutairi, A. Nanogels from Metal-Chelating Crosslinkers as Versatile Platforms Applied to Copper-64 PET Imaging of Tumors and Metastases. Theranostics 2015, 5, 277-288.

(186) Krasia-Christoforou, T.; Georgiou, T. K. Polymeric Theranostics: using Polymer-Based Systems for Simultaneous Imaging and Therapy. J. Mater. Chem. B 2013, 1, 3002-3025.

(187) Mauri, E.; Veglianese, P.; Papa, S.; Mariani, A.; De Paola, M.; Rigamonti, R.; Chincarini, G. M. F.; Vismara, I.; Rimondo, S.; Sacchetti, A.; Rossi, F. Double Conjugated Nanogels for Selective Intracellular Drug Delivery. RSC Adv. 2017, 7, 30345-30356.

(188) Mauri, E.; Moroni, I.; Magagnin, L.; Masi, M.; Sacchetti, A.; Rossi, F. Comparison between Two Different Click Strategies to Synthesize Fluorescent Nanogels for Therapeutic Applications. React. Funct. Polym. 2016, 105, 35-44.

(189) Aktan, B.; Chambre, L.; Sanyal, R.; Sanyal, A. "Clickable" Nanogels via Thermally Driven Self-Assembly of Polymers: Facile Access to Targeted Imaging Platforms using Thiol-Maleimide Conjugation. Biomacromolecules 2017, 18, 490-497.

(190) Park, J. S.; Yi, S. W.; Kim, H. J.; Kim, S. M.; Shim, S. H.; Park, K.H. Sunflower-Type Nanogels Carrying a Quantum Dot Nanoprobe for both Superior Gene Delivery Efficacy and Tracing of Human Mesenchymal Stem Cells. Biomaterials 2016, 77, 14-25.

(191) Saha, A.; Devi, P. S. Surface Functionalized Multifunctional Gd2O3-Fluorescein Composite Nanorods for Redox Responsive Drug Delivery and Imaging Applications. ACS Appl. Nano Mater. 2018, $1,2898-2911$.

(192) Guo, H.; Li, F. P.; Xu, W. G.; Chen, J. J.; Hou, Y. C.; Wang, C. X.; Ding, J. X.; Chen, X. S. Mucoadhesive Cationic Polypeptide Nanogel with Enhanced Penetration for Efficient Intravesical Chemotherapy of Bladder Cancer. Adv. Sci. 2018, 5, 1800004.

(193) Yang, W. J.; Zhou, P.; Liang, L. J.; Cao, Y. P.; Qiao, J. Q.; Li, X. H.; Teng, Z. G.; Wang, L. H. Nanogel-Incorporated Injectable Hydrogel for Synergistic Therapy Based on Sequential Local Delivery of Combretastatin-A4 Phosphate (CA4P) and Doxorubicin (DOX). ACS Appl. Mater. Interfaces 2018, 10, 18560-18573.

(194) Panonnummal, R.; Jayakumar, R.; Anjaneyan, G.; Sabitha, M. In Vivo Anti-Psoriatic Activity, Biodistribution, Sub-acute and Subchronic Toxicity Studies of Orally Administered Methotrexate Loaded Chitin Nanogel in Comparison with Methotrexate Tablet. Int. J. Biol. Macromol. 2018, 110, 259-268.

(195) Panonnummal, R.; Sabitha, M. Anti-Psoriatic and Toxicity Evaluation of Methotrexate Loaded Chitin Nanogel in Imiquimod Induced Mice Model. Int. J. Biol. Macromol. 2018, 110, 245-258. 